\title{
Introducing the CTA Concept
}

B.S. Acharya ${ }^{\text {bc }}$, M. Actis ${ }^{f}$, T. Aghajani ${ }^{\text {dx }}$, G. Agnetta ${ }^{\text {bi }}$, J. Aguilared A. F. Aharonian $^{\text {bd }}$,

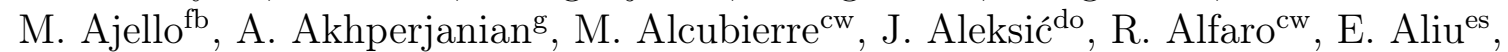
A.J. Allafort ${ }^{\mathrm{fb}}$, D. Allan ${ }^{\text {eg }}$, I. Allekotte ${ }^{\mathrm{c}}$, E. Amato ${ }^{\mathrm{bj}}$, J. Anderson ${ }^{\text {er }}$, E.O. Angüner ${ }^{\mathrm{al}}$, L.A. Antonelli bo, P. Antoranz ${ }^{\mathrm{dt}}$, A. Aravantinos ${ }^{\mathrm{ay}}$, T. Arlen ${ }^{\mathrm{fd}}$, T. Armstrong ${ }^{\mathrm{eg}}$,

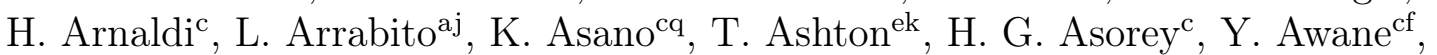
H. Baba ${ }^{\text {by }}$, A. Babic ${ }^{\mathrm{t}}$, N. Baby ${ }^{\mathrm{ae}}$, J. Bähr ${ }^{\text {aw }}$, A. Bais ${ }^{\text {ax }}$, C. Baixeras ${ }^{\text {dv }}$, S. Bajtlik ${ }^{\text {de }}$, M. Balbo ${ }^{\text {ee }}$,D. Balis ${ }^{\text {ax }}$, C. Balkowski ${ }^{a k}$, A. Bamba ${ }^{\text {bv }}$, R. Bandiera ${ }^{\text {bj }}$, A. Barber ${ }^{f j}$, C. Barbier ${ }^{\text {ag }}$, M. Barceló ${ }^{\text {do }}$, A. Barnackade ${ }^{\text {de }}$ J. Barnstedt ${ }^{\text {au }}$, U. Barres de Almeida ${ }^{j}$, J.A. Barrio $^{\text {ds }}$, A. Basilied ${ }^{\text {ed S. Basso }}{ }^{\text {bl }}$, D. Bastieri ${ }^{\text {bs }}$, C. Bauer ${ }^{\text {aq }}$, A. Baushev ${ }^{\text {at }}$,

J. Becerra ${ }^{\text {dr,ap}}$, Y. Becherini ${ }^{\mathrm{ab}}$, K.C. Bechtol ${ }^{\mathrm{fb}}$, J. Becker Tjus ${ }^{\mathrm{am}}$, V. Beckmann ${ }^{\mathrm{ab}}$,

W. Bednarek ${ }^{\text {di }}$, B. Behera ${ }^{\text {aw }}$, M. Belluso ${ }^{\text {bm }}$, W. Benbow ${ }^{\text {ev }}$, J. Berdugo ${ }^{\text {dn }}$, K. Berger $^{\text {dr }}$, F. Bernard ${ }^{\text {eb }}$, T. Bernardino ${ }^{d t}$, K. Bernlöhraq ${ }^{\text {aq }}$ N. Bhat ${ }^{\text {ba }}$, S. Bhattacharyya ${ }^{\text {ba }}$, C. Bigongiari ${ }^{\text {bp }}$, A. Biland ${ }^{\mathrm{ec}}$, S. Billotta ${ }^{\mathrm{bm}}$, T. Bird ${ }^{\mathrm{ep}}$, E. Birsin ${ }^{\text {aw }, a l}$, E. Bissaldi ${ }^{\mathrm{h}}$, J. Biteau ${ }^{\text {ah }}$, M. Bitossi ${ }^{\text {bt }}$, S. Blake ${ }^{\text {ek }}$, O. Blanch Bigas ${ }^{\text {do }}$, P. Blasi ${ }^{\text {bj }}$, A. Bobkov ${ }^{\text {fd }}$, V. Boccone ${ }^{\mathrm{ed}}$, M. Boettcher ${ }^{\mathrm{dl}}$, L. Bogacz ${ }^{\mathrm{dd}}$, J. Bogart ${ }^{\mathrm{fb}}$, M. Bogdan ${ }^{\mathrm{ff}}$, C. Boisson ${ }^{\mathrm{ak}}$, J. Boix Gargallo ${ }^{\text {do }}$, J. Bolmont ${ }^{\text {ai }}$, G. Bonanno ${ }^{\text {bm }}$, A. Bonardia ${ }^{\text {au }}$ T. Bonev ${ }^{\mathrm{q}}$,

P. Bonifacio ${ }^{\text {ak }}$, G. Bonnoli ${ }^{\text {bl }}$, P. Bordas ${ }^{\text {au }}$, A. Borgland ${ }^{\mathrm{fb}}$, J. Borkowski ${ }^{\mathrm{de}}$, R. Bose ${ }^{\mathrm{fk}}$,

O. Botner ${ }^{\mathrm{ea}}$, A. Bottani ${ }^{\mathrm{f}}$, L. Bouchet ${ }^{\mathrm{ae}}$, M. Bourgeat ${ }^{\mathrm{aj}}$, C. Boutonnet $^{\mathrm{ab}}$, A. Bouvier $^{\mathrm{fa}}$,

S. Brau-Noguéae, I. Braun ${ }^{\text {ec }}$, T. Bretz ${ }^{\text {eb,ee }}$, M. Briggs ${ }^{\text {fc }}$, T. Bringmann ${ }^{\text {ap }}$, P. Brook ${ }^{\text {en }}$, P. Brun ${ }^{\mathrm{af}}$, L. Brunetti ${ }^{\mathrm{ag}}$, T. Buanes ${ }^{\mathrm{da}}$, J. Buckley ${ }^{\mathrm{fk}}$, R. Buehler ${ }^{\mathrm{aw}}$, V. Bugaev ${ }^{\mathrm{fk}}$,

A. Bulgarellibf ${ }^{\text {bf }}$ T. Bulik ${ }^{\text {dj }}$, G. Busetto ${ }^{\text {bs }}$, S. Buson ${ }^{\text {bs }}$, K. Byrum ${ }^{\text {er }}$, M. Cailles ${ }^{\text {ag }}$, R. Cameron ${ }^{\text {fb }}$, J. Camprecios ${ }^{\text {dp }}$, R. Canestrari ${ }^{\text {bl }}$, S. Cantu ${ }^{\text {bl }}$, M. Capalbi ${ }^{\text {bo }}$, P. Caraveo ${ }^{\text {bg }}$, E. Carmona ${ }^{\text {dn }}$, A. Carosi ${ }^{\text {bo }}$, J. Carr ${ }^{\text {ac }}$, P.-H. Carton ${ }^{\text {af }}$, S. Casanova ${ }^{\text {aq,dl, }}$, M. Casiraghi ${ }^{\text {bl }}$, O. Catalano ${ }^{\text {bi }}$, S. Cavazzani ${ }^{\text {bn }}$, S. Cazaux ${ }^{\text {af }}$, M. Cerruti ${ }^{\text {ev }}$, E. Chabanne ${ }^{\text {ag }}$, P. Chadwick ${ }^{\text {eg }}$, C. Champion ${ }^{\mathrm{ab}}$, A. Chen ${ }^{\mathrm{bg}}$, J. Chiang ${ }^{\mathrm{fb}}$, L. Chiappetti ${ }^{\mathrm{bg}}$, M. Chikawa ${ }^{\mathrm{cb}}$, V.R. Chitnis ${ }^{\mathrm{bc}}$, F. Chollet ${ }^{\mathrm{ag}}$, J. Chudobaw ${ }^{\mathrm{w}}$, M. Cieślar ${ }^{\mathrm{dj}}$, A. Cillis ${ }^{\mathrm{d}}$, J. Cohen-Tanugi ${ }^{\mathrm{aj}}$,

S. Colafrancesco ${ }^{\mathrm{dm}}$, P. Colin ${ }^{\mathrm{as}}$, J. Colome ${ }^{\mathrm{dp}}$, S. Colonges $^{\text {ab }}$, M. Compin $^{\text {aj }}$, P. Conconi ${ }^{\mathrm{bl}}$, V. Conforti ${ }^{\text {bf }}$, V. Connaughton ${ }^{\text {fc }}$, J. Conrad ${ }^{\text {dz }}$, J.L. Contreras ${ }^{\text {ds }}$, P. Coppi ${ }^{\text {fl }}$, P. Corona ${ }^{\text {ai }}$,

D. Corti ${ }^{\text {bs }}$, J. Cortina ${ }^{\text {do }}$, L. Cossio ${ }^{\text {bu }}$, H. Costantini ${ }^{a c}$, G. Cotter en, B. Courty ${ }^{\text {ab }}$,

S. Couturier ${ }^{\text {ah }}$, S. Covino ${ }^{\text {bl }}$, G. Crimi ${ }^{\text {bl }}$, S.J. Criswellev, J. Croston ${ }^{\text {ep }}$, G. Cusumano ${ }^{\text {bi }}$,

M. Dafonseca ${ }^{\text {ae }}$, O. Dale ${ }^{\text {da }}$, M. Daniel ${ }^{\text {eg }}$, J. Darling ${ }^{\mathrm{el}}$, I. Davids ${ }^{\mathrm{cx}}$, F. Dazzi ${ }^{\mathrm{bs}}$,

A. De Angelis ${ }^{\text {bu }}$, V. De Caprio ${ }^{\text {bg }}$, F. De Frondat ${ }^{\text {ak }}$, E.M. de Gouveia Dal Pino ${ }^{\text {, }}$

I. de la Calle ${ }^{\text {ds }}$, G.A. De La Vega ${ }^{b}$, R. de los Reyes Lopez ${ }^{\mathrm{aq}}$, B. De Lotto ${ }^{\text {bu }}$, A. De Luca ${ }^{\mathrm{bg}}$,

J.R.T. de Mello Neton ${ }^{n}$ M. de Naurois ${ }^{\text {ah }}$, Y. de Oliveira ${ }^{\text {ah }}$, E. de Oña Wilhelmidp,

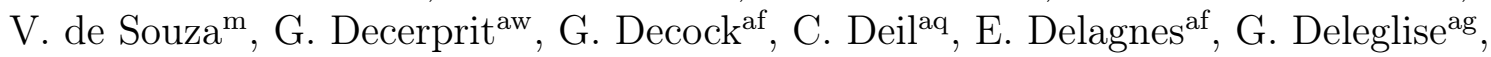

C. Delgado ${ }^{\text {dn }}$, D. Della Volpe ${ }^{\text {ed }}$, P. Demange ${ }^{\text {ak }}$, G. Depaola $^{\mathrm{d}}$, A. Dettlaff ${ }^{\text {as }}$,

A. Di Paola ${ }^{\text {bo }}$,F. Di Pierro ${ }^{\text {bp }}$, C. Díaz ${ }^{\text {dn }}$, J. Dick ${ }^{\mathrm{au}}$, R. Dickherber ${ }^{\mathrm{fk}}$, H. Dickinson ${ }^{\mathrm{dz}}$, V. Diez-Blanco ${ }^{\text {aq }}$, S. Dige ${ }^{\mathrm{fb}}$, D. Dimitrov ${ }^{\mathrm{q}}$, G. Disset ${ }^{\mathrm{af}}$, A. Djannati-Atai ${ }^{\mathrm{ab}}$, M. Doert $^{\mathrm{an}}$,

M. Dohmke an, W. Domainko aq, D. Dominis Prester ${ }^{\mathrm{u}}$, A. Donat ${ }^{\mathrm{aw}}$, D. Dorner $^{\mathrm{ee}}$,

M. Doro ${ }^{\text {dv }}$, J.-L. Dournaux ${ }^{\text {ak }}$, G. Drake ${ }^{\text {er }}$, D. Dravins ${ }^{\mathrm{dx}}$, L. Drury ${ }^{\mathrm{bd}}$, F. Dubois ${ }^{\mathrm{ds}}$,

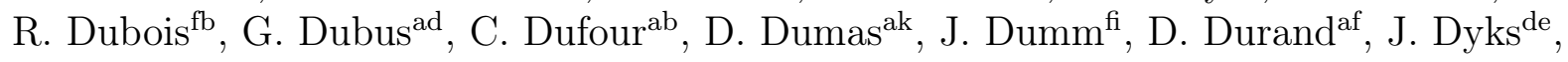




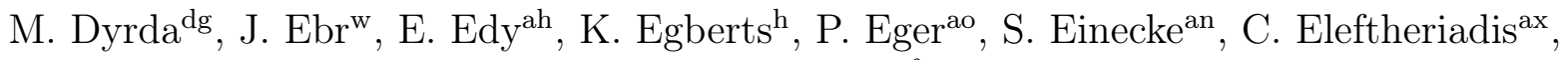
S. Elles ${ }^{\text {ag }}$, D. Emmanoulopoulos ${ }^{\text {ep }}$, D. Engelhaupt ${ }^{\text {fc }}$, R. Enomoto ${ }^{\text {cs }}$, J.-P. Ernenwein ${ }^{\text {ac }}$, M. Errando ${ }^{\text {es }}$, A. Etchegoyen ${ }^{\text {b }}$, P. Evans ${ }^{\text {ek }}$, A. Falcone ${ }^{\text {ex }}$, D. Fantinel ${ }^{\text {bn }}$, K. Farakos ${ }^{\text {ay }}$, C. Farnieree,dz, G. Fasola ${ }^{\text {ak }}$, B. Favillek ${ }^{\text {ek }}$ E. Fede ${ }^{\text {ag }}$, S. Federici ${ }^{\text {at }}$, S. Fegan ${ }^{\text {ah }}$,

F. Feinstein ${ }^{\text {aj }}$, D. Ferenc ${ }^{\text {fe }}$, P. Ferrando ${ }^{\text {af }}$, M. Fesquet ${ }^{\text {af }}$, A. Fiasson $^{\text {ag }}$, E. Fillin-Martino $^{\text {ah }}$, D. Fink ${ }^{\mathrm{as}}$, C. Finley ${ }^{\mathrm{dz}}$, J. P. Finley ${ }^{\mathrm{ez}}$, M. Fiorinibs, R. Firpo Curcoll ${ }^{\mathrm{do}}$, H. Flores ${ }^{\mathrm{ak}}$,

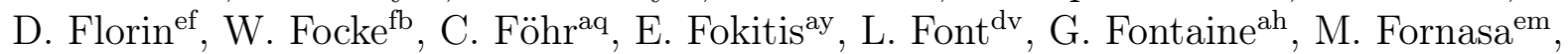
A. Förster ${ }^{\mathrm{aq}}$, L. Fortson ${ }^{\mathrm{fi}}$, N. Fouque ${ }^{\mathrm{ag}}$, A. Franckowiak ${ }^{\mathrm{fb}}$, C. Fransson ${ }^{\mathrm{dz}}$, G. Fraser ${ }^{\mathrm{ek}}$, R. Frei ${ }^{\mathrm{eb}}$, I.F.M. Albuquerque ${ }^{\mathrm{l}}$, L. Fresnillo ${ }^{\mathrm{dt}}$, C. Fruck $^{\mathrm{as}}$, Y. Fujita ${ }^{\mathrm{cl}}$, Y. Fukazawa ${ }^{\text {bx }}$, Y. Fukui ${ }^{\text {cj }}$, S. Funk ${ }^{\text {fb }}$, W. Gäbele ${ }^{\text {au }}$, S. Gabici ${ }^{\text {ab }}$, R. Gabriele ${ }^{\text {bn }}$, A. Gadola ${ }^{\text {ef }}$, N. Galante ev D. Gall ${ }^{\mathrm{fh}}$, Y. Gallant ${ }^{\mathrm{aj}}$, J. Gámez-García ${ }^{\mathrm{du}}$, B. García ${ }^{\mathrm{b}}$, R. Garcia López ${ }^{\mathrm{dr}}$, D. Gardiol ${ }^{\text {bp }}$, D. Garrido ${ }^{\text {dv }}$, L. Garrido ${ }^{\text {dw }}$, D. Gascon ${ }^{\text {dw }}$, M. Gaug ${ }^{\text {dv }}$, J. Gaweda ${ }^{\text {do }}$,

L. Gebremedhin ${ }^{\text {fa }}$, N. Geffroy ${ }^{\text {ag }}$, L. Gerard ${ }^{\text {aw }}$, A. Ghedina ${ }^{\text {br }}$, M. Ghigo ${ }^{\text {bl }}$,

E. Giannakakiax, F. Gianottibf ${ }^{\text {bf }}$, S. Giarrusso ${ }^{\text {bi }}$, G. Giavitto ${ }^{\text {do }}$, B. Giebels ${ }^{\text {ah }}$, V. Gika ${ }^{\text {ay }}$, P. Giommi ${ }^{\text {bo }}$, N. Girard ${ }^{\text {ae }}$, E. Giro ${ }^{\text {bn }}$, A. Giuliani ${ }^{\text {bg }}$, T. Glanzman ${ }^{\text {fb }}$, J.-F. Glicenstein ${ }^{\text {af }}$, N. Godinovic ${ }^{\mathrm{s}}$, V. Golev ${ }^{\mathrm{r}}$, M. Gomez Berisso ${ }^{\mathrm{c}}$, J. Gómez-Ortega ${ }^{\mathrm{du}}$, M.M. Gonzalez $^{\mathrm{cw}}$,

A. González ${ }^{\text {do }}$, F. González ${ }^{\mathrm{a}}$, A. González Muñoz ${ }^{\mathrm{do}}$, K.S. Gothe ${ }^{\mathrm{bc}}$, M. Gougerot ${ }^{\mathrm{ag}}$, R. Graciani ${ }^{\text {dw }}$, P. Grandi ${ }^{\text {bf }}$, F. Grañena ${ }^{\text {do }}$, J. Granot ${ }^{\mathrm{ej}}$, G. Grasseau ${ }^{\text {ah }}$, R. Gredigef,

A. Green ${ }^{\mathrm{em}}$, T. Greenshaw ${ }^{\mathrm{el}}$, T. Grégoire ${ }^{\mathrm{ae}}$, O. Grimm ${ }^{\mathrm{ec}}$, J. Grube ${ }^{\mathrm{eq}}$, M. Grudzinska ${ }^{\mathrm{dj}}$, V. Gruev ${ }^{\text {fk }}$, S. Grünewald ${ }^{\text {aw }}$, J. Grygorczuk ${ }^{\mathrm{df}}$, V. Guarino ${ }^{\mathrm{er}}$, S. Gunji ${ }^{\mathrm{cu}}$, G. Gyuk ${ }^{\mathrm{eq}}$, D. Hadasch ${ }^{\text {dp }}$, R. Hagiwara ${ }^{\text {cu }}$, J. Hahn $^{\text {aq }}$, N. Hakansson ${ }^{\text {at }}$, A. Hallgren ${ }^{\text {ea }}$,

N. Hamer Heras ${ }^{\text {dn }}$, S. Hara ${ }^{\text {cv }}$, M.J. Hardcastle ${ }^{\mathrm{ej}}$, J. Harris ${ }^{\mathrm{eg}}$, T. Hassan ${ }^{\text {ds }}$, K. Hatanaka ${ }^{\text {cf }}$,

T. Haubold ${ }^{\text {as }}$, A. Haupt ${ }^{\text {aw }}$, T. Hayakawa ${ }^{\text {cj }}$, M. Hayashida ${ }^{\text {ce }}$, R. Heller ${ }^{\text {aw }}$, F. Henault ${ }^{\text {ad }}$, G. Henria ${ }^{\text {ad }}$, G. Hermann ${ }^{\text {aq }}$, R. Hermel ${ }^{\text {ag }}$, A. Herrero ${ }^{\text {dr }}$, N. Hidaka ${ }^{\text {ck }}$, J. Hinton ${ }^{\text {ek}}$,

D. Hoffmann ${ }^{\text {ac }}$, W. Hofmann ${ }^{\text {aq }}$, P. Hofverbergaq, J. Holder ${ }^{\text {fg }}$, D. Horns ${ }^{\text {ap }}$, D. Horville ${ }^{\text {ak }}$, J. Houles ${ }^{\text {ac }}$, M. Hrabovsky ${ }^{\mathrm{w}}$, D. Hrupec ${ }^{\mathrm{t}}$, H. Huan ${ }^{\mathrm{ff}}$, B. Huber ${ }^{\text {ef }}$, J.-M. Huet ${ }^{\mathrm{ak}}$,

G. Hughes ${ }^{\text {aw }}$, T.B. Humensky ${ }^{\text {et }}$, J. Huovelin ${ }^{y}$, A. Ibarra ${ }^{\text {ds }}$, J.M. Illa ${ }^{\text {do }}$, D. Impiombato ${ }^{\text {bi }}$,

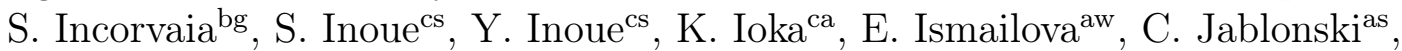

A. Jacholkowska ${ }^{\text {ai }}$, M. Jamrozy ${ }^{\text {dd }}$, M. Janiak ${ }^{\text {de }}$, P. Jean ${ }^{\text {ae }}$, C. Jeanney ${ }^{\text {af }}$, J.J. Jimenez ${ }^{\text {dt }}$, T. Jogler ${ }^{\text {fb }}$, T. Johnson ${ }^{\text {fb }}$, L. Journet ${ }^{\text {ag }}$, C. Juffroy ${ }^{\text {ab }}$, I. Jung ${ }^{\text {ao }}$, P. Kaaret ${ }^{\text {fh }}$, S. Kabuki ${ }^{\text {co }}$, M. Kagaya $^{\text {by }}$, J. Kakuwa ${ }^{\text {bx }}$, C. Kalkuhl ${ }^{\text {au }}$, R. Kankanyan ${ }^{\text {aq }}$, A. Karastergiou ${ }^{\text {en }}$,

K. Kärcher ${ }^{\text {ao }}$, M. Karczewski ${ }^{\text {df }}$, S. Karkar ${ }^{\text {ac }}$, J. Kasperek ${ }^{\text {dc }}$, D. Kastana ${ }^{\text {ay }}$, H. Katagiri ${ }^{\text {by }}$, J. Kataoka ${ }^{\text {ct }}$, K. Katarzyński ${ }^{\text {dh }}$, U. Katz ${ }^{\text {ao }}$, N. Kawanaka ${ }^{\text {cs }}$, B. Kellner-Leidel ${ }^{\text {aw }}$, H. Kelly ${ }^{\mathrm{fb}}$, E. Kendziorra ${ }^{\text {au }}$, B. Khélifi ${ }^{\text {ah }}$, D.B. Kieda ${ }^{\mathrm{fj}}$, T. Kifune ${ }^{\mathrm{cs}}$, T. Kihm ${ }^{\mathrm{aq}}$, T. Kishimoto ${ }^{\text {cf }}$, K. Kitamoto ${ }^{\text {cb }}$, W. Kluźniak ${ }^{\text {de }}$, C. Knapic ${ }^{\text {bq }}$, J. Knapp ${ }^{\text {aw }}$, J. Knödlseder ${ }^{\text {ae }}$, F. Köck ${ }^{\mathrm{aq}}$, J. Kocot ${ }^{\mathrm{db}}$, K. Kodani ${ }^{\mathrm{cn}}$, J.-H. Köhne ${ }^{\text {an }}$, K. Kohri ${ }^{\mathrm{ca}}$, K. Kokkotas ${ }^{\text {au,ax }}$, D. Kolitzus ${ }^{\text {h }}$, N. Komin ${ }^{\text {ag }}$, I. Kominis ${ }^{\text {ay }}$, Y. Konno ${ }^{\text {cf }}$, H. Köppel ${ }^{\text {aw }}$, P. Korohodadc , K. Kosack ${ }^{\text {af }}$, G. Koss ${ }^{\text {aw }}$, R. Kossakowski ${ }^{\text {ag }}$, P. Kostka ${ }^{\text {aw }}$, R. Koul ${ }^{\text {ba }}$, G. Kowal ${ }^{\mathrm{k}}$, S. Koyama ${ }^{\mathrm{cm}}$, J. Kozioł ${ }^{\mathrm{dd}}$, T. Krähenbühl ${ }^{\mathrm{ec}}$, J. Krause ${ }^{\text {as }}$, H. Krawzcynski ${ }^{\text {fk }}$,

F. Krennrich ${ }^{\text {ew }}$, A. Krepps ${ }^{\text {er }}$, A. Kretzschmann ${ }^{\text {aw }}$, R. Krobot ${ }^{\text {ao }}$, P. Krueger ${ }^{\text {aq,dl }}$, H. Kubo ${ }^{\text {cf }}$, V.A. Kudryavtsev ${ }^{\text {eo }}$, J. Kushida ${ }^{\text {cn }}$, A. Kuznetsov ${ }^{\text {fa }}$, A. La Barbera ${ }^{\text {bi }}$, N. La Palombara ${ }^{\text {bg }}$, V. La Parola ${ }^{\text {bi }}$, G. La Rosa ${ }^{\text {bi }}$, K. Lacombe Le $^{\text {ae }}$ G. Lamanna ${ }^{\text {ag }}$, J. Lande $^{\mathrm{fb}}$, D. Languignon ${ }^{\mathrm{ak}}$, J. Lapington ${ }^{\mathrm{ek}}$, P. Laporte ${ }^{\mathrm{ak}}$, C. Lavalley $^{\mathrm{aj}}$, T. Le Flour ${ }^{\mathrm{ag}}$, 
A. Le Padellec ${ }^{\text {ae }}$, S.-H. Lee ${ }^{\text {cg }}$, W.H. Lee ${ }^{\text {cw }}$, M.A. Leigui de Oliveira ${ }^{\mathrm{i}}$, D. Lelas ${ }^{\mathrm{s}}$, J.-P. Lenain ${ }^{\mathrm{ai}}$, D.J. Leopold ${ }^{\mathrm{fk}}$, T. Lerch $^{\text {af }}$, L. Lessio ${ }^{\mathrm{bn}}$, B. Lieunard ${ }^{\mathrm{ag}}$, E. Lindfors ${ }^{\mathrm{aa}}$, A. Liolios ${ }^{\text {ax }}$, A. Lipniackada ${ }^{\text {da }}$ H. Lockart ${ }^{\mathrm{fd}}$, T. Lohse ${ }^{\mathrm{al}}$, S. Lombardi ${ }^{\text {bo }}$, A. Lopatin ${ }^{\text {ao }}$, M. Lopez ${ }^{\text {ds }}$, R. López-Coto ${ }^{\text {do }}$, A. López-Oramas ${ }^{\text {do }}$, A. Lorca $^{\text {ds }}$, E. Lorenz $^{\text {as }}$, P. Lubinski ${ }^{\mathrm{de}}$, F. Lucarelli ${ }^{\text {bo }}$, H. Lüdecke ${ }^{\mathrm{aw}}$, J. Ludwin ${ }^{\mathrm{dg}}$, P.L. Luque-Escamilladu,

W. Lustermann ${ }^{\mathrm{ec}}$, O. Luz ${ }^{\mathrm{au}}$, E. Lyard ${ }^{\mathrm{ee}}$, M.C. Maccarone ${ }^{\mathrm{bi}}$, T.J. Maccarone ${ }^{\mathrm{ep}}$, G.M. Madejskii ${ }^{\mathrm{fb}}$, A. Madhavan ${ }^{\mathrm{ew}}$, M. Mahabir ${ }^{\mathrm{ek}}$, G. Maier ${ }^{\mathrm{aw}}$, P. Majumdar ${ }^{\mathrm{bb}}$, G. Malaguti bf , S. Maltezos ${ }^{\text {ay }}$, A. Manalaysay ${ }^{\text {ef }}$, A. Mancilla ${ }^{\mathrm{b}}$, D. Mandat ${ }^{\mathrm{w}}$, G. Maneva ${ }^{\mathrm{p}}$, A. Mangano ${ }^{\text {bi }}$, P. Manigot ${ }^{\text {ah }}$, K. Mannheim ${ }^{\text {av }}$, I. Manthos ${ }^{\text {ay }}$, N. Maragos ${ }^{\text {ay }}$, A. Marcowith ${ }^{\text {aj }}$, M. Mariotti ${ }^{\text {bs }}$, M. Marisaldi ${ }^{\text {bf }}$, S. Markoff ${ }^{\text {cy }}$, A. Marszałek ${ }^{\text {dd }}$, C. Martens ${ }^{\mathrm{aw}}$, J. Martídu, J.-M. Martinak ${ }^{\mathrm{ak}}$ P. Martin ${ }^{\mathrm{ae}}$, G. Martínez ${ }^{\mathrm{dn}}$, F. Martínez ${ }^{\mathrm{dp}}$, M. Martínez ${ }^{\text {do }}$, A. Masserot ${ }^{\text {ag }}$, A. Mastichiadis $^{\text {az }}$, A. Mathieu $^{\text {ah }}$, H. Matsumoto ${ }^{\text {ci }}$, F. Mattana ${ }^{\text {ab }}$, S. Mattiazzo ${ }^{\text {bs }}$, G. Maurin ${ }^{\mathrm{ag}}$, S. Maxfield ${ }^{\mathrm{el}}$, J. Maya ${ }^{\mathrm{b}}$, D. Mazin ${ }^{\mathrm{as}}$, L. Mc Comb ${ }^{\mathrm{eg}}$, N. McCubbin ${ }^{\mathrm{eh}}$, I. McHardy ${ }^{\mathrm{ep}}, \mathrm{R} . \mathrm{McKay}^{\mathrm{ew}}, \mathrm{C} . \mathrm{Medina}^{\mathrm{e}}$, C. Meliolik ${ }^{\mathrm{k}}$, D. Melkumyan ${ }^{\text {aw }}$, S. Mereghetti ${ }^{\text {bg }}$, P. Mertsch ${ }^{\text {en }}$, M. Meucci ${ }^{\text {bt }}$, J. Michałowskidg, P. Micolon ${ }^{\text {af }}$, A. Mihailidis ${ }^{\text {ax }}$, T. Mineo ${ }^{\text {bi }}$, M. Minuti ${ }^{\text {bt }}$, N. Mirabal ${ }^{\text {ds }}$, F. Mirabel $^{\text {af }}$, J.M. Miranda ${ }^{\mathrm{dt}}$, R. Mirzoyan ${ }^{\mathrm{as}}$, T. Mizuno ${ }^{\mathrm{bw}}$, B. Moal ${ }^{\mathrm{ah}}$, R. Moderski ${ }^{\mathrm{de}}$, I. Mognet ${ }^{\mathrm{fd}}$, E. Molinari $^{\text {br }}$, M. Molinaro ${ }^{\text {bf }}$, T. Montarulied ${ }^{\text {, I. Monteiro }}{ }^{\text {ag }}$, P. Moore ${ }^{\text {fk }}$,

A. Moralejo Olaizola ${ }^{\text {do }}$, M. Mordalskadf ${ }^{\text {df }}$ C. Morello ${ }^{\text {bp }}$, K. Mori ${ }^{\text {ch }}$, F. Mottez ${ }^{\text {ak }}$, Y. Moudden ${ }^{\text {af }}$, E. Moulin ${ }^{\text {af }}$, I. Mrusek ${ }^{\text {aw }}$, R. Mukherjee ${ }^{\mathrm{es}}$, P. Munar-Adrover ${ }^{\mathrm{dw}}$, H. Muraishicc ${ }^{\text {cc }}$ K. Murase ${ }^{\text {cs }}$, A. Murphy ${ }^{\text {ei }}$, S. Nagatakic ${ }^{\text {cg }}$, T. Naito ${ }^{\text {cv }}$, D. Nakajima ${ }^{\text {as,cs }}$, T. Nakamori ${ }^{\mathrm{ct}}$, K. Nakayama ${ }^{\mathrm{cr}}$, C. Naumann ${ }^{\mathrm{ai}}$, D. Naumann ${ }^{\mathrm{aw}}$, M. Naumann-Godo ${ }^{\text {ab }}$, P. Nayman ${ }^{\text {ai }}$, D. Nedbal ${ }^{\text {}}$, D. Neise ${ }^{\text {an }}$, L. Nellen ${ }^{\text {cw }}$, V. Neustroev ${ }^{\mathrm{z}}$, N. Neyroud $^{\text {ag }}$, L. Nicastro ${ }^{\text {bf }}$, J. Nicolau-Kukliński ${ }^{\text {df }}$, A. Niedźwiecki ${ }^{\text {di }}$, J. Niemiec ${ }^{\text {dg }}$, D. Nieto ${ }^{\text {et }}$, A. Nikolaidis ${ }^{\text {ax }}$, K. Nishijima ${ }^{\text {cn }}$, S. Nolan ${ }^{\text {eg }}$, R. Northrop ${ }^{\text {ff }}$, D. Nosek ${ }^{\mathrm{v}}$, N. Nowak ${ }^{\text {as }}$, A. Nozato ${ }^{\text {cb }}$, P. O'Brien ${ }^{\text {ek }}$, Y. Ohira ${ }^{\text {bv }}$, M. Ohishics ${ }^{\text {cs }}$ S. Ohm ${ }^{\text {ek }}$, H. Ohoka ${ }^{\text {cs }}$, T. Okuda ${ }^{\text {cj }}$, A. Okumura ${ }^{\text {ck }}$, J.-F. Olive ${ }^{\text {ae }}$, R.A. Ong ${ }^{\mathrm{fd}}$, R. Orito ${ }^{\mathrm{cp}}$, M. Orr ${ }^{\mathrm{ew}}$, J. Osborne ${ }^{\mathrm{ek}}$, M. Ostrowski ${ }^{\mathrm{dd}}$, L.A. Otero ${ }^{\mathrm{a}}$, N. Otte ${ }^{\mathrm{eu}}$, E. Ovcharov ${ }^{\mathrm{r}}$, I. Oya ${ }^{\mathrm{al}}$, A. Ozieblo $^{\mathrm{db}}$, L. Padilla ${ }^{\text {ds }}$, S. Paiano ${ }^{\text {bs }}$, D. Paillot ${ }^{\text {ab }}$, A. Paizis ${ }^{\text {bg }}$, S. Palanque ${ }^{\text {af }}$, M. Palatkaw ${ }^{\text {, }}$ J. Pallota ${ }^{\text {a }}$, K. Panagiotidis ${ }^{\text {ax }}$, J.-L. Panazol ${ }^{\text {ag }}$, D. Paneque ${ }^{\text {as }}$, M. Panter ${ }^{\text {aq }}$, R. Paoletti ${ }^{\text {bt }}$, A. Papayannis ${ }^{\text {ay }}$, G. Papyan ${ }^{\text {g }}$, J.M. Paredes ${ }^{\mathrm{dw}}$, G. Pareschi ${ }^{\mathrm{bl}}$, G. Parks ${ }^{\mathrm{fa}}$, J.-M. Parraud ${ }^{\text {ai }}$, D. Parsons ${ }^{\mathrm{aq}}$, M. Paz Arribas ${ }^{\mathrm{al}}$, M. Pech ${ }^{\mathrm{w}}$, G. Pedalettid ${ }^{\mathrm{dp}}$, V. Pelassa ${ }^{\mathrm{fc}}$,

D. Pelat ${ }^{\text {ak }}$, M. d. C. Perez ${ }^{\mathrm{dt}}$, M. Persic ${ }^{\text {bq }}$, P.-O. Petrucci ${ }^{\text {ad }}$, B. Peyaud ${ }^{\text {af }}$, A. Pichel ${ }^{\mathrm{d}}$, S. Pita ${ }^{\text {ab }}$, F. Pizzolato ${ }^{\text {bg }}$, Ł. Platos ${ }^{\text {df }}$, R. Platzer ${ }^{\text {aw }}$, L. Pogosyan ${ }^{g}$, M. Pohl $^{\text {at }}$, G. Pojmanski ${ }^{\text {dj }}$, J.D. Ponz ${ }^{\text {ds }}$, W. Potter ${ }^{\text {en }}$, J. Poutanen ${ }^{z}$, E. Prandinibs, J. Prast ${ }^{\text {ag }}$, R. Preece ${ }^{\text {eh }}$, F. Profeti ${ }^{\text {bt }}$, H. Prokoph ${ }^{\text {aw }}$, M. Prouza ${ }^{w}$, M. Proyettia ${ }^{\mathrm{a}}$, I. Puerto-Gimenez ${ }^{\mathrm{dr}}$, G. Pühlhofer ${ }^{\text {au }}$, I. Puljak ${ }^{\mathrm{s}}$, M. Punch ${ }^{\mathrm{ab}}$, R. Pyzioł ${ }^{\mathrm{dg}}$, E.J. Quel ${ }^{\mathrm{a}}$, J. Quinn ${ }^{\mathrm{be}}$, A. Quirrenbach ${ }^{\text {ar }}$, E. Racero ${ }^{\mathrm{ds}}$, P.J. Rajda ${ }^{\mathrm{dc}}$, P. Ramon ${ }^{\mathrm{ae}}$, R. Rando ${ }^{\mathrm{bs}}$, R.C. Rannot ${ }^{\mathrm{ba}}$, M. Rataj ${ }^{\mathrm{df}}$, M. Raue ${ }^{\mathrm{ap}}$, P. Reardon ${ }^{\mathrm{fc}}$, O. Reimann ${ }^{\mathrm{as}}$, A. Reimer ${ }^{\mathrm{h}}$, O. Reimer ${ }^{\mathrm{h}}$, K. Reitberger ${ }^{\text {h }}$ M. Renaud ${ }^{\text {aj }}$, S. Renner ${ }^{\text {au }}$, B. Reville ${ }^{\text {en }}$, W. Rhode ${ }^{\text {an }}$, M. Ribó $^{\text {dw }}$, M. Ribordy ${ }^{\mathrm{eb}}$, M.G. Richer ${ }^{\mathrm{cw}}$, J. Rico ${ }^{\mathrm{do}}$, J. Ridky ${ }^{\mathrm{w}}$, F. Rieger ${ }^{\mathrm{aq}}$, P. Ringegni ${ }^{\mathrm{f}}$, J. Ripken ${ }^{\mathrm{dz}}$, P.R. Ristori ${ }^{\mathrm{a}}$, A. Rivière ${ }^{\mathrm{ac}}$, S. Rivoire ${ }^{\mathrm{aj}}$, L. Rob ${ }^{\mathrm{v}}$, U. Roeser ${ }^{\mathrm{ec}}$, R. Rohlfs ${ }^{\mathrm{ee}}$, G. Rojas ${ }^{\mathrm{o}}$, P. Romano ${ }^{\text {bi }}$, W. Romaszkan ${ }^{\mathrm{dc}}$, G. E. Romero ${ }^{\mathrm{e}}$, S. Rosen ${ }^{\mathrm{ek}}$, S. Rosier Lees $^{\mathrm{ag}}$, 
D. Ross ${ }^{\mathrm{ek}}$, G. Rouaix ${ }^{\mathrm{ae}}$, J. Rousselle $^{\mathrm{fd}}$, S. Rousselle ${ }^{\mathrm{cy}}$, A.C. Rovero $^{\mathrm{d}}$, F. Roy $^{\mathrm{ak}}$, S. Royer ${ }^{\mathrm{aj}}$, B. Rudak ${ }^{\mathrm{de}}$, C. Rulten ${ }^{\mathrm{eg}}$, M. Rupińskidc ${ }^{\mathrm{dc}}$ F. Russo ${ }^{\text {bi }}$, F. Ryde ${ }^{\mathrm{dy}}$, B. Sacco ${ }^{\mathrm{bi}}$, E.O. Saemann ${ }^{\text {aw }}$, A. Saggion ${ }^{\text {bs }}$, V. Sahakian ${ }^{\text {g }}$, K. Saito ${ }^{\text {cs }}$, T. Saito ${ }^{\text {as }}$, Y. Saito ${ }^{\text {cn }}$, N. Sakaki ${ }^{\text {cs }}$, R. Sakonaka ${ }^{\text {cb }}$, A. Salinibl ${ }^{\text {bl }}$ F. Sanchez ${ }^{\text {b }}$, M. Sanchez-Conde ${ }^{\mathrm{fb}}$, A. Sandoval ${ }^{\mathrm{cw}}$, H. Sandaker ${ }^{\text {da }}$, E. Sant'Ambrogio ${ }^{\text {bg }}$, A. Santangelo ${ }^{\text {au }}$, E.M. Santos ${ }^{\text {n }}$, A. Sanuy ${ }^{\text {dw }}$, L. Sapozhnikov ${ }^{\mathrm{fb}}$, S. Sarkar ${ }^{\mathrm{en}}$, N. Sartore ${ }^{\mathrm{bg}}$, H. Sasaki ${ }^{\mathrm{cd}}$, K. Sataleckads, M. Sawada ${ }^{\mathrm{bv}}$, V. Scalzotto ${ }^{\text {bs }}$, V. Scapin ${ }^{\text {ds }}$, M. Scarcioffolo ${ }^{\text {bs }}$, J. Schafer ${ }^{\text {h }}$, T. Schanz ${ }^{\text {au }}$, S. Schlenstedt ${ }^{\text {aw }}$, R. Schlickeiser ${ }^{\text {am }}$, T. Schmidt ${ }^{\text {aw }}$, J. Schmoll ${ }^{\mathrm{eg}}$, P. Schovanek ${ }^{\mathrm{w}}$, M. Schroedter ${ }^{\mathrm{ev}}$, C. Schultz ${ }^{\text {bs }}$, J. Schultze ${ }^{\text {aw }}$, A. Schulz ${ }^{\text {aw }}$, K. Schure ${ }^{\text {en }}$, T. Schwabaq, U. Schwanke ${ }^{\text {al }}$, J. Schwarz ${ }^{\text {bl }}$, S. Schwarzburgau, T. Schweizer ${ }^{\text {as }}$, S. Schwemmer ${ }^{\text {ar }}$, A. Segreto ${ }^{\text {bi }}$, J.-H. Seiradakis ${ }^{a x}$, G.H. Sembroskiez, K. Seweryn ${ }^{\text {df }}$, M. Sharmaba, M. Shayduk ${ }^{\text {aw }}$, R.C. Shellard ${ }^{j}$, J. Shi ${ }^{\text {fd }}$, T. Shibata ${ }^{\text {bv }}$, A. Shibuyack ${ }^{\text {ck }}$ E. Shum ${ }^{\mathrm{eg}}$, L. Sidoli ${ }^{\text {bg }}$, M. Sidz ${ }^{\mathrm{df}}$, J. Sieiro ${ }^{\text {dw }}$, M. Sikora ${ }^{\text {de }}$, J. Silk ${ }^{\text {en }}$, A. Sillanpääaa ${ }^{\text {aa }}$ B.B. Singh ${ }^{\text {bc }}$, J. Sitarek ${ }^{\text {di,do }}$, C. Skole ${ }^{\text {aw }}$, R. Smareglia ${ }^{\text {bq }}$, A. Smith ${ }^{\mathrm{fj}}$, D. Smith ${ }^{\mathrm{fb}}$, J. Smith ${ }^{\mathrm{fj}}$, N. Smith ${ }^{\mathrm{ey}}$, D. Sobczyńskadi, H. Solak ${ }^{\text {ak }}$ G. Sottile ${ }^{\text {bi }}$, M. Sowińskidg, F. Spanier ${ }^{\text {av }}$, D. Spiga ${ }^{\text {bl }}$, S. Spyrou ${ }^{\text {ah }}$, V. Stamatescudo, A. Stamerra ${ }^{\text {bp }}$, R. Starlingek ${ }^{\text {ek }}$ Ł. Stawarz ${ }^{\text {dd }}$, R. Steenkamp ${ }^{\text {cx }}$, C. Stegmann ${ }^{\text {aw }}$, S. Steiner ${ }^{\text {ef }}$, N. Stergioulas ${ }^{\text {ax }}$, R. Sternberger ${ }^{\text {aw }}$, M. Sterzel ${ }^{\text {db }}$, F. Stinzing ${ }^{\text {ao }}$, M. Stodulskidg,

U. Straumann ${ }^{\text {ef }}$, E. Strazzeri ${ }^{\text {bi }}$, L. Stringhettibg, A. Suarez ${ }^{\mathrm{e}}$, M. Suchenek ${ }^{\mathrm{dj}}$,

R. Sugawara ${ }^{\text {cp }}$, K.-H. Sulanke ${ }^{\text {aw }}$, S. Sun ${ }^{\text {as }}$, A.D. Supanitsky ${ }^{\text {d }}$, T. Suric ${ }^{\mathrm{t}}$, P. Sutcliffe ${ }^{\mathrm{el}}$, J. Sykes ${ }^{\mathrm{ek}}$, M. Szanecki ${ }^{\mathrm{di}}$, T. Szepieniec ${ }^{\mathrm{db}}$, A. Szostek ${ }^{\mathrm{dd}}$, G. Tagliaferri ${ }^{\mathrm{bl}}$, H. Tajima ${ }^{\mathrm{ck}}$,

H. Takahashi ${ }^{\text {bx }}$, K. Takahashicj, L. Takalo ${ }^{\text {aa }}$, H. Takami ${ }^{\text {ca }}$, G. Talboteg, J. Tammi ${ }^{\text {, }}$, M. Tanaka ${ }^{\text {ca }}$, S. Tanakaby ${ }^{\text {by }}$ J. Tasan ${ }^{\text {ag }}$, M. Tavani ${ }^{\text {bh }}$, J.-P. Tavernet ${ }^{\text {ai }}$, L.A. Tejedor ${ }^{\text {ds }}$,

I. Telezhinsky ${ }^{\text {at }}$, P. Temnikov ${ }^{\mathrm{p}}$, C. Tenzer ${ }^{\mathrm{au}}, \mathrm{Y}$. Terada ${ }^{\mathrm{cm}}, \mathrm{R}$. Terrier ${ }^{\mathrm{ab}}$, M. Teshima ${ }^{\mathrm{as}, \mathrm{cs}}$, V. Testa ${ }^{\text {bo }}$, D. Tezier ${ }^{\text {ac }}$, D. Thuermann ${ }^{\text {aw }}$, L. Tibaldo ${ }^{\text {bs,fb }}$, O. Tibolla ${ }^{\text {av }}$, A. Tiengo ${ }^{\text {bg }}$, M. Tluczykont ${ }^{\text {ap }}$, C.J. Todero Peixoto ${ }^{\mathrm{m}}$, F. Tokanai ${ }^{\mathrm{cu}}$, M. Tokarz ${ }^{\mathrm{df}}$, K. Toma ${ }^{\mathrm{cl}}$, K. Torii ${ }^{\mathrm{cj}}$, M. Tornikoski ${ }^{\mathrm{x}}$, D.F. Torres ${ }^{\mathrm{dq}}$, M. Torres ${ }^{\mathrm{cw}}$, G. Tosti ${ }^{\mathrm{bl}}$, T. Totani $^{\mathrm{ce}}$,

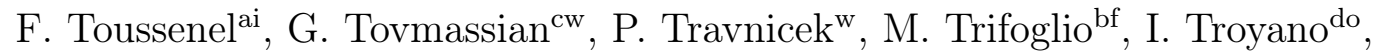

K. Tsinganos ${ }^{\text {az }}$, H. Ueno ${ }^{\mathrm{cm}}$, K. Umehara ${ }^{\text {by }}$, S.S. Upadhya ${ }^{\text {bc }}$, T. Usher ${ }^{\mathrm{fb}}$, M. Uslenghi $^{\mathrm{bg}}$, J.F. Valdes-Galicia ${ }^{\text {cw }}$, P. Vallania ${ }^{\text {bp }}$, G. Vallejo ${ }^{f}$, W. van Driel ${ }^{\text {ak }}$, C. van Eldik ${ }^{\mathrm{ao}}$, J. Vandenbrouke ${ }^{\mathrm{fb}}$, J. Vanderwalt ${ }^{\mathrm{dl}}$, H. Vankov ${ }^{\mathrm{p}}$, G. Vasileiadis ${ }^{\mathrm{aj}}$, V. Vassiliev ${ }^{\mathrm{fd}}$, D. Veberic ${ }^{\mathrm{dk}}$, I. Vegas $^{\mathrm{dt}}$, S. Vercellone $^{\mathrm{bi}}$, S. Vergani $^{\mathrm{ak}}$, C. Veyssière ${ }^{\mathrm{af}}$, J.P. Vialle $^{\mathrm{ag}}$,

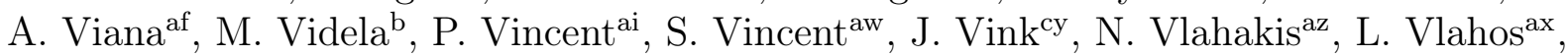
P. Voglerec , A. Vollhardtef ${ }^{\text {ef }}$ H.-P. von Gunten ${ }^{\text {ec }}$, S. Vorobiov ${ }^{\text {aw }}$, C. Vuerli ${ }^{\text {bq }}$,

V. Waegebaert ${ }^{\text {ae }}$, R. Wagner ${ }^{\text {as }}$, R.G. Wagner ${ }^{\mathrm{er}}$, S. Wagner ${ }^{\text {ar }}$, S.P. Wakely ${ }^{\mathrm{ff}}$, R. Walter ${ }^{\mathrm{ee}}$, T. Walther ${ }^{\text {aw }}$, K. Warda ${ }^{\text {an }}$, R. Warwick ${ }^{\text {ek }}$, P. Wawer ${ }^{\text {df }}$, R. Wawrzaszek ${ }^{\text {df }}$, N. Webb ${ }^{\text {ae }}$, P. Wegner ${ }^{\text {aw }}$, A. Weinstein ${ }^{\text {ew }}$, Q. Weitzel ${ }^{\text {aq }}$, R. Welsing ${ }^{\text {aw }}$, M. Werner ${ }^{\text {h }}$, H. Wetteskind ${ }^{\text {as }}$, R. White ${ }^{\mathrm{ek}}$, A. Wierzcholska ${ }^{\mathrm{dd}}$, S. Wiesand ${ }^{\text {aw }}$, M. Wilkinson $^{\mathrm{ek}}$, D.A. Williams ${ }^{\mathrm{fa}}$, R. Willingale ${ }^{\mathrm{ek}}, \mathrm{K}$. Winiarski ${ }^{\mathrm{dc}}, \mathrm{R}$. Wischnewski ${ }^{\mathrm{aw}}$, Ł. Wiśniewski $^{\mathrm{df}}, \mathrm{M}$. Wood ${ }^{\mathrm{fb}}$, A. Wörnlein ${ }^{\text {ao }}$, Q. Xiong ${ }^{\text {ey }}$, K.K. Yadav ${ }^{\text {ba }}$, H. Yamamoto ${ }^{\text {cj }}$, T. Yamamoto ${ }^{\text {cd }}$, R. Yamazaki ${ }^{\text {bv }}$, S. Yanagita ${ }^{\text {by }}$, J.M. Yebras ${ }^{\text {dt }}$, D. Yelos ${ }^{\text {b }}$, A. Yoshida ${ }^{\text {bv }}$, T. Yoshida ${ }^{\text {by }}$, T. Yoshikoshics, V. Zabalza ${ }^{\text {dw }}$, M. Zachariass ${ }^{\text {am }}$, A. Zajczyk ${ }^{\text {de }}$, R. Zanin ${ }^{\text {dw }}$, A. Zdziarski ${ }^{\text {de }}$, A. Zech ${ }^{\text {ak }}$, A. Zhao ${ }^{\text {er }}$, X. Zhou ${ }^{\text {cb }}$, K. Ziętara ${ }^{\text {dd }}$, J. Ziolkowskide, P. Ziółkowskidg, V. Zitellibk ${ }^{\text {bk }}$ C. Zurbach ${ }^{\text {aj }}$, P. Żychowski ${ }^{\mathrm{dg}}$ 
${ }^{a}$ Centro de Investigaciones en Láseres y Aplicaciones (CEILAP - CITEFA / CONICET), Argentina

${ }^{b}$ Instituto de Tecnologias en Deteccion y Astroparticulas (CNEA / CONICET / UNSAM), Argentina ${ }^{c}$ Centro Atómico Bariloche (CNEA-CONICET-IB/UNCUYO), Bariloche, Argentina

${ }^{d}$ Instituto de Astronomía y Física del Espacio (CONICET-UBA), Buenos Aires, Argentina

e Instituto Argentino de Radioastronomía (CCT La Plata - CONICET), La Plata, Argentina

${ }^{f}$ UID GEMA - Departamento de Aeronáutica (Facultad de Ingeniería, UNLP), La Plata, Argentina ${ }^{g}$ Alikhanyan National Science Laboratory, Yerevan, Armenia

${ }^{h}$ Institut für Astro- und Teilchenphysik, Leopold-Franzens-Universität, Innsbruck, Austria

${ }^{i}$ Centro de Ciências Naturais e Humanas, Universidade Federal do ABC, Brazil ${ }^{j}$ Centro Brasileiro de Pesquisas Fúsicas, Rio de Janeiro, Brazil

${ }^{k}$ Instituto de Astronomia, Geofísico, e Ciências Atmosféricas, Universidade de São Paulo, Brazil ${ }^{l}$ Instituto de Física, Universidade de São Paulo, Brazil

${ }^{m}$ Instituto de Física de São Carlos, Universidade de São Paulo, Brazil

${ }^{n}$ Instituto de Física, Universidade Federal do Rio de Janeiro, Brazil

o Núcleo de Formação de Professores, Universidade Federal de São Carlos, Brazil

${ }^{p}$ Institute for Nuclear Research and Nuclear Energy, BAS, Sofia, Bulgaria

${ }^{q}$ Institute of Astronomy, BAS, Sofia, Bulgaria

${ }^{r}$ Astronomy Department of Faculty of Physics, Sofia University, Sofia, Bulgaria

${ }^{s}$ FESB - University of Split, Croatia

${ }^{t}$ Rudjer Boskovic Institute, Zagreb, Croatia

${ }^{u}$ University of Rijeka, Physics Department, Rijeka, Croatia

${ }^{v}$ Charles University, Institute of Particle and Nuclear Physics, Prague, Czech Republic

${ }^{w}$ Institute of Physics of the Academy of Sciences of the Czech Republic, Prague, Czech Republic

${ }^{x}$ Aalto University, Finland

${ }^{y}$ University of Helsinki, Finland

${ }^{z}$ Department of Physics, University of Oulu, Finland

${ }^{a}$ Tuorla Observatory, University of Turku, Finland

${ }^{a b}$ APC, Univ Paris Diderot, CNRS/IN2P3, CEA/lrfu, Obs de Paris, Sorbonne Paris Cité, France

${ }^{a c}$ Centre de Physique des Particules de Marseille (CPPM), Aix-Marseille Université, CNRS/IN2P3, Marseille, France

${ }^{a d}$ Institut de Planétologie et d'Astrophysique de Grenoble, INSU/CNRS, Université Joseph Fourier, Gernoble, France

ae Institut de Planétologie et d'Astrophysique (IRAP), CNRS, Université Paul Sabatier, Toulouse, France ${ }^{\text {af } C E A / D S M / I R F U, \text { Saclay, France }}$

${ }^{a g}$ Laboratoire d'Annecy-le-Vieux de Physique des Particules, Université de Savoie, CNRS/IN2P3, Annecy, France

${ }^{a h}$ Laboratoire Leprince-Ringuet, École Polytechnique (UMR 7638, CNRS), Paris, France

${ }^{a i}$ LPNHE, University of Pierre et Marie Curie, Paris 6, University of Denis Diderot, Paris 7, CNRS/IN2P3, Paris, France

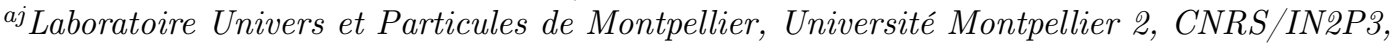
Montpellier, France

${ }^{a k}$ Observatoire de Paris, LUTH and GEPI, CNRS, Université Paris Diderot, Paris, France

${ }^{a l}$ Institut für Physik, Humboldt-Universität zu Berlin, Germany

${ }^{a m}$ Institut für Theoretische Physik, Lehrstuhl IV: Weltraum- und Astrophysik, Ruhr-Universität Bochum, Germany

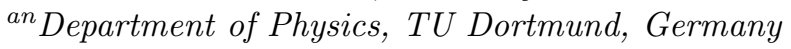

${ }^{a o}$ Universität Erlangen-Nürnberg, Physikalisches Institut, Erlangen, Germany

${ }^{a p}$ Universität Hamburg, Institut für Experimentalphysik, Hamburg, Germany ${ }^{a q}$ Max-Planck-Institut für Kernphysik, Heidelberg, Germany

${ }^{a r}$ Landessternwarte, Universität Heidelberg, Germany

${ }^{a s}$ Max-Planck-Institut für Physik, München, Germany

${ }^{a t}$ Institut für Physik and Astronomie, Universität Potsdam, Germany 
${ }^{a u}$ Institut für Astronomie und Astrophysik, Universität Tübingen, Germany

${ }^{a v}$ Institute for Theoretical Physics and Astrophysics, Universität Würzburg, Germany

${ }^{a w}$ Deutsches Elektronen-Synchrotron, Platanenallee 6, Zeuthen, Germany

${ }^{a x}$ School of Physics, Aristotle University, Thessaloniki, Greece

${ }^{a y}$ National Technical University of Athens, Department of Physics, Athens, Greece

${ }^{a z}$ Faculty of Physics, National and Kapodestrian University of Athens, Greece

${ }^{b a}$ Bhabha Atomic Research Centre (BARC), India

${ }^{b b}$ Saha Institute of Nuclear Physics, India

${ }^{b c}$ Tata Institute of Fundamental Research (TIFR), Mumbai, India

${ }^{b d}$ Dublin Institute for Advanced Studies, Ireland

be University College Dublin, Ireland

${ }^{b f}$ INAF - Istituto di Astrofisica Spaziale e Fisica Cosmica di Bologna, Italy

${ }^{b g}$ INAF - Istituto di Astrofisica Spaziale e Fisica Cosmica di Milano, Italy

${ }^{b h}$ INAF - Istituto di Astrofisica Spaziale e Fisica Cosmica di Roma, Italy

${ }^{b i} I N A F$ - Istituto di Astrofisica Spaziale e Fisica Cosmica di Palermo, Italy

${ }^{b j} I N A F$ - Osservatorio Astrofisico di Arcetri, Firenze, Italy

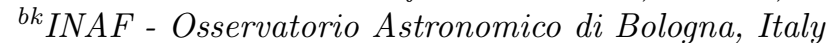

${ }^{b l} I N A F$ - Osservatorio Astronomico di Brera, Italy

${ }^{b m}$ INAF - Osservatorio Astrofisico di Catania, Italy

${ }^{b n}$ INAF - Osservatorio Astronomico di Padova, Italy

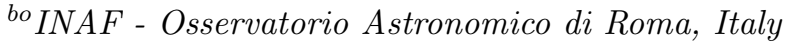

${ }^{b p}$ INAF - Osservatorio Astrofisico di Torino, Italy

${ }^{b q}$ INAF - Osservatorio Astronomico di Trieste and INFN Sezione di Trieste, Italy

${ }^{b r}$ INAF - Telescopio Nazionale Galileo, Roma, Italy

${ }^{b s}$ Dipartimento di Fisica, Universitá degli Studi di Padova, Italy

${ }^{b t}$ Università di Siena and INFN, Siena, Italy

${ }^{b u}$ University of Udine and INFN Sezione di Trieste, Italy

${ }^{b v}$ Department of Physics and Mathematics, Aoyama Gakuin University, Japan

${ }^{b w}$ Hiroshima Astrophysical Science Center, Hiroshima University, Japan

${ }^{b x}$ Department of Physical Science, Hiroshima University, Japan

${ }^{b y}$ Faculty of Science, Ibaraki University, Japan

${ }^{b z}$ Institute of Space and Astronautical Science, JAXA, Japan

${ }^{c a}$ Institute of Particle and Nuclear Studies, KEK (High Energy Accelerator Research Organization), Japan

${ }^{c b}$ Dept. of Physics, Kinki University, Japan

${ }^{c c}$ School of Allied Health Sciences, Kitasato University, Japan

${ }^{c d}$ Department of Physics, Konan University, Japan

${ }^{c e}$ Department of Astronomy, Kyoto University, Japan

${ }^{c f}$ Department of Physics, Kyoto University, Japan

${ }^{c g}$ Yukawa Institute for Theoretical Physics, Kyoto University, Japan

${ }^{c h}$ Department of Applied Physics, University of Miyazaki, Japan

${ }^{c i}$ Kobayashi-Maskawa Institute (KMI) for the Origin of Particles and the Universe, Nagoya University, Japan

${ }^{c j}$ Department of Physics and Astrophysics, Nagoya University, Japan

${ }^{c k}$ Solar-Terrestrial Environment Laboratory, Nagoya University, Japan

${ }^{c l}$ Department of Earth and Space Science, Graduate School of Science, Osaka University, Japan

${ }^{c m}$ Graduate School of Science and Engineering, Saitama University, Japan

${ }^{c n}$ Department of Physics, Tokai University, Japan

co Tokai University Hospital, Japan

${ }^{c p}$ Institute of Socio-Arts and Sciences, University of Tokushima, Japan

${ }^{c q}$ Interactive Research Center of Science, Tokyo Institute of Technology, Tokyo, Japan

${ }^{c r}$ Department of Physics, Graduate School of Science, University of Tokyo, Tokyo, Japan 
${ }^{c s}$ Institute for Cosmic Ray Research, University of Tokyo, Tokyo, Japan

${ }^{c t}$ Faculty of Science and Engineering, Waseda University, Japan ${ }^{c u}$ Department of Physics, Yamagata University, Japan

${ }^{c v}$ Faculty of Management Information, Yamanashi Gakuin University, Japan

${ }^{c w}$ Universidad Nacional Autónoma de México, Mexico City, Mexico

${ }^{c x}$ University of Namibia, Department of Physics, Namibia

${ }^{c y}$ Astronomical Institute Anton Pannekoek, University of Amsterdam, Netherlands

${ }^{c z}$ Radboud University Nijmegen, Netherlands

${ }^{d a}$ Department of Physics and Technology, University of Bergen, Norway

${ }^{d b}$ Academic Computer Centre CYFRONET AGH, Kraków, Poland

${ }^{d c}$ Faculty of Electrical Engineering, Automatics, Computer Science and Electronics, AGH University of Science and Technology, Kraków, Poland

${ }^{d d}$ Faculty of Physics, Astronomy and Applied Computer Science, Jagiellonian University, Poland

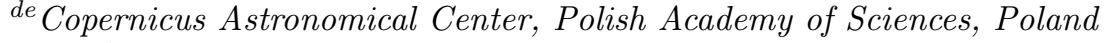

${ }^{d f}$ Space Research Centre, Polish Academy of Sciences, Poland

${ }^{d g}$ Institute of Nuclear Physics, Polish Academy of Sciences, Poland

${ }^{d h}$ Torun Centre for Astronomy, Nicolaus Copernicus University, Poland

${ }^{d i}$ Faculty of Physics and Applied Computer Science, University of Lódż, Poland

${ }^{d j}$ Faculty of Physics, University of Warsaw, Poland

${ }^{d k}$ Laboratory for Astroparticle Physics, University of Nova Gorica, Slovenia

${ }^{d l}$ Centre for Space Research, North-West University, Potchefstroom, South Africa

${ }^{d m}$ School of Physics, University of the Witwatersrand, Johannesburg, South Africa

${ }^{d n}$ CIEMAT, Madrid, Spain

do Institut de Física d'Altes Energies, Barcelona, Spain

${ }^{d p}$ Institut de Ciències de l'Espai (IEEC-CSIC), Barcelona, Spain

${ }^{d q}$ Institut de Ciències de l'Espai (IEEC-CSIC) and Institució Catalana de Recerca I Estudis Avancats (ICREA), Spain

${ }^{d r}$ Instituto de Astrofísica de Canarias, Spain

${ }^{d s}$ Grupo de Altas Energías. Universidad Complutense de Madrid, Spain

${ }^{d t}$ Grupo de Electronica, Universidad Complutense de Madrid, Spain

${ }^{d u}$ Escuela Politécnica Superior de Jaén, Universidad de Jaén, Spain

${ }^{d v}$ Departament de Física, and CERES-IEEC, Universitat Autònoma de Barcelona, E-08193 Bellaterra, Spain

${ }^{d w}$ Departament d'Astronomia i Meteorologia, Institut de Ciències del Cosmos (ICC), Universitat de Barcelona (IEEC-UB), Spain

${ }^{d x}$ Lund Observatory, Lund, Sweden

${ }^{d y}$ Oskar Klein Centre, Department of Physics, Royal Institute of Technology (KTH), Stockholm, Sweden ${ }^{d z}$ Stockholm University, Stockholm, Sweden

${ }^{e a}$ Dept. of Physics and Astronomy, Uppsala University, Sweden

${ }^{e b}$ Laboratory for High Energy Physics, École Polytechnique Fédérale, Switzerland

${ }^{e c}$ ETH Zürich, Institute for Particle Physics, Switzerland

ed University of Geneva - Département de Physique Nucléaire et Corpusculaire, Switzerland

${ }^{e e}$ ISDC Data Centre for Astrophysics, Observatory of Geneva, University of Geneva, Switzerland

ef Physik-Institut, Universität Zürich, Switzerland

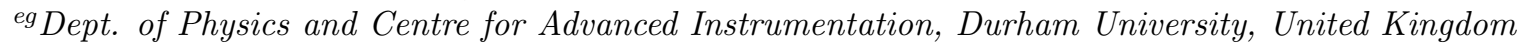

${ }^{e h}$ STFC Rutherford Appleton Laboratory, United Kingdom

${ }^{e i}$ School of Physics and Astronomy, University of Edinburgh, United Kingdom

${ }^{e j}$ Centre for Astrophysics Research, Science and Technology Research Institute, University of Hertfordshire, United Kingdom

${ }^{e k}$ Dept. of Physics and Astronomy, University of Leicester, United Kingdom

${ }^{e l}$ University of Liverpool, Oliver Lodge Laboratory, Liverpool, United Kingdom

${ }^{e m}$ School of Physics and Astronomy, University of Nottingham, United Kingdom 


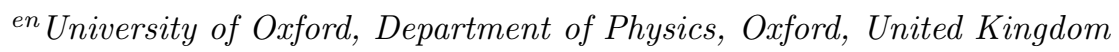

${ }^{e o}$ Department of Physics and Astronomy, University of Sheffield, Sheffield, United Kingdom

ep School of Physics and Astronomy, University of Southampton, United Kingdom

${ }^{e q}$ Astronomy Department, Adler Planetarium and Astronomy Museum, Chicago, USA

er Argonne National Laboratory, USA

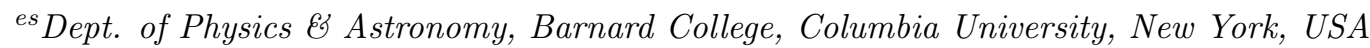

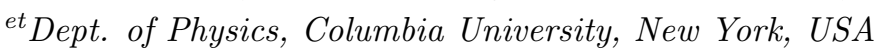

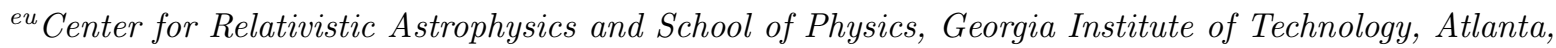
$U S A$

${ }^{e v}$ Harvard-Smithsonian Center for Astrophysics, Cambridge, USA

ew Department of Physics and Astronomy, Iowa State University, USA

ex Dept. of Astronomy and Astrophysics, Pennsylvania State University, USA

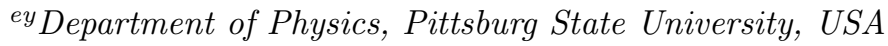

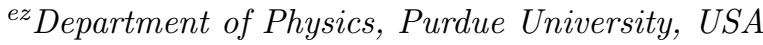

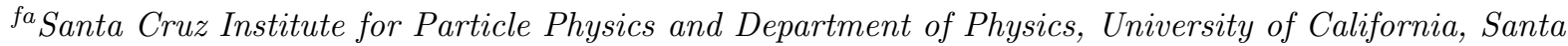
Cruz, USA

${ }^{f b}$ Kavli Institute for Particle Astrophysics and Cosmology, Department of Physics and SLAC National Accelerator Laboratory, Stanford University, USA

${ }^{f c}$ University of Alabama in Huntsville, Center for Space Physics and Aeronomic Research, Huntsville, $U S A$

${ }^{f d}$ Department of Physics and Astronomy, University of California, Los Angeles, USA

fe University of California, Davis, USA

${ }^{f f}$ Enrico Fermi Institute, University of Chicago, Chicago, USA

${ }^{f g}$ Department of Physics and Astronomy and the Bartol Research Institute, University of Delaware, Newark, USA

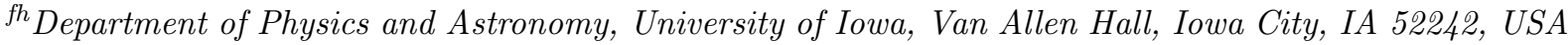

${ }^{f i}$ School of Physics and Astronomy, University of Minnesota, Minneapolis, USA

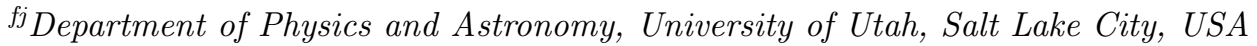

${ }^{f k}$ Department of Physics, Washington University, St Louis, USA

${ }^{f l}$ Yale University, Department of Physics and Astronomy, New Haven, USA

\begin{abstract}
The Cherenkov Telescope Array (CTA) is a new observatory for very high-energy (VHE) gamma rays. CTA has ambitions science goals, for which it is necessary to achieve fullsky coverage, to improve the sensitivity by about an order of magnitude, to span about four decades of energy, from a few tens of $\mathrm{GeV}$ to above $100 \mathrm{TeV}$ with enhanced angular and energy resolutions over existing VHE gamma-ray observatories. An international collaboration has formed with more than 1000 members from 27 countries in Europe, Asia, Africa and North and South America. In 2010 the CTA Consortium completed a Design Study and started a three-year Preparatory Phase which leads to production readiness of CTA in 2014. In this paper we introduce the science goals and the concept of CTA, and provide an overview of the project.
\end{abstract}




\section{Introduction}

Very high-energy $\left(\mathrm{VHE}^{1}\right)$ electromagnetic radiation reaches Earth from a large part of the Cosmos, carrying crucial and unique information about the most energetic phenomena in the Universe. Yet, it has only been in the last 25 years that we have had instruments to "see" this radiation. The situation changed with the development of imaging air Cherenkov telescopes (IACTs) [1] and air shower detectors, which have now matured to open a new window for exploration of the high-energy Universe. Current IACT instruments, such as the H.E.S.S. [2], MAGIC [3] and VERITAS [4] telescope systems, together with the very successful Fermi [5] and AGILE [6] satellites and the air shower experiments Milagro [7], Tibet AS-gamma [8] and ARGO-YBJ [9], which have been designed for gamma-ray detection, have produced a wealth of exciting results and have demonstrated that VHE phenomena are ubiquitous throughout the Universe $[10,11]$. But many of the results have raised new questions which require more and better data for a deeper understanding of the underlying phenomena.

CTA [12] will answer many of the persisting questions by enabling the detection of more than 1000 sources over the whole sky [10]. CTA builds on the proven technique of detecting gamma-ray induced particle cascades in the atmosphere through their Cherenkov radiation, simultaneously imaging each cascade stereoscopically with multiple telescopes, and reconstructing the properties of the primary gamma ray from those images. Through deployment of about 50-100 telescopes per site at two sites in the southern and the northern hemispheres CTA will achieve full-sky coverage. This number of telescopes, which will come in three sizes, improves the sensitivity and the energy coverage by at least an order of magnitude compared to existing VHE instruments. Also, the angular and energy resolutions will improve significantly, yielding unparalleled imaging capability at very high energies. In addition, the improved sensitivity may permit the discovery of completely new and unexpected phenomena. For the first time in this energy range, CTA will be operated from the outset as an open observatory. CTA will accept observing proposals from interested scientists and provide tools and support for data analysis.

The CTA concept was first proposed to the ESFRI ${ }^{2}$ committee in 2006 as research infrastructure for gamma-ray astronomy with an estimated investment cost of around 150 $\mathrm{M} €($ at 2006 cost levels). The CTA Consortium formed to design the instrument and to work towards its implementation. Since then the interest and the support for the project have grown, and currently the CTA Consortium consists of more than 1000 scientists and engineers in more than 160 institutions from 27 countries around the globe.

This article presents the scope and concept of CTA in section 2. The main scientific drivers are outlined in section 3 and, in much greater detail, in the other papers in this Topical Issue. In section 4 the technological concepts of the CTA baseline design are presented and in section 5 the expected performance for that design is discussed. Section 6 is devoted to the observatory aspects of the CTA project. Finally, section 7 summarises the status and the plans for the project.

\footnotetext{
${ }^{1}>100 \mathrm{GeV}$

${ }^{2}$ European Strategy Forum on Research Infrastructures
} 


\section{CTA Scope and Concept}

The latest generation of ground-based gamma-ray instruments have enabled the imaging, photometry and spectroscopy of VHE gamma-ray sources and have propelled their studies into a genuine branch of astronomy. The number of known VHE gamma-ray sources currently exceeds 150, and source types include supernova remnants (SNRs), pulsars and pulsar wind nebulae, binary stellar systems, interacting stellar winds, various types of active galaxies, and unidentified sources without any obvious counterparts in other wavelength ranges. H.E.S.S. has conducted a highly successful survey of the Milky Way covering about 600 square degrees, which resulted in the detection of tens of new sources [13]. A similarly sensitive survey of the full visible sky with current instruments would require at least a decade of observations, and is therefore unrealistic.

Due to the low flux of VHE photons (even at some tens of $\mathrm{GeV}$ ), detectors for these energies require a large detection area, effectively ruling out space-borne instruments that directly detect the incident gamma rays. Ground-based instruments have much larger effective detection areas than the typical size of $1 \mathrm{~m}^{2}$ of detectors flown in space. Groundbased gamma-ray detectors detect the particle cascades induced when VHE gamma rays interact in the atmosphere, either by recording shower particles reaching arrays of detectors at ground or mountain altitudes, or by using Cherenkov telescopes to image the shower in Cherenkov light which is emitted by secondary charged particles in the cascade. The effective area is then at least the size of the shower-particle or Cherenkov-light footprint on the ground, which is of the order of $10^{5} \mathrm{~m}^{2}$.

Compared to Cherenkov telescopes, air shower detectors such as Tibet AS-gamma or ARGO-YBJ have the advantage of a large duty cycle, as they can also observe during daytime, and of large instantaneous sky coverage. However, despite observing times of years, their sensitivities allow currently only detection of sources approximately as bright as the Crab nebula, the strongest steady source of VHE gamma rays. Results from air shower detectors show that there are relatively few sources emitting at this level. Therefore, the recent rapid evolution of ground-based gamma-ray astronomy was primarily driven by atmospheric Cherenkov telescope arrays. They reach sensitivities of $1 \%$ of the Crab flux for observing times of about $25 \mathrm{~h}$ and have significantly better angular and energy resolutions. Projects to develop air shower detectors with much improved sensitivity are underway (e.g. the HAWC project [14], in construction), which will offer valuable complementary information, but they will not be able to compete in sensitivity and resolution with arrays of imaging Cherenkov telescopes such as CTA.

At lower energies there will be overlap with the energy range of the satellite instruments, contemporaneous or even simultaneous observations with the Fermi Large Area Telescope will be possible. The Fermi all-sky catalog will provide many sources for CTA to target and while Fermi observations complement CTA down to MeV energies, CTA will have a sensitivity for short-timescale phenomena that is orders of magnitude better than that of Fermi.

The properties of the major current and previous air Cherenkov instruments are listed in table 1. The current telescope arrays consist of 2-5 Cherenkov telescopes. They reach sensitivities of about $1 \%$ of the Crab flux at energies in the $0.1-1 \mathrm{TeV}$ range. Sensitivity 
degrades towards lower energies, due to insufficient background rejection, and towards higher energies, due to the limited number of gamma rays. A typical angular resolution is $0.1^{\circ}$ or slightly better for a single gamma ray, but sufficiently intense point sources can be located with a precision of $10-20$ arc seconds.

\begin{tabular}{|c|c|c|c|c|c|c|c|c|c|}
\hline Instrument & $\begin{array}{l}\text { Lat } \\
\left({ }^{\circ}\right)\end{array}$ & $\begin{array}{l}\text { Long } \\
\left(^{\circ}\right)\end{array}$ & $\begin{array}{l}\text { Alt } \\
(\mathrm{m})\end{array}$ & & $\begin{array}{l}\text { Area } \\
\left(\mathrm{m}^{2}\right)\end{array}$ & $\begin{array}{c}\text { Pixels per } \\
\text { camera }\end{array}$ & $\begin{array}{l}\mathrm{FoV} \\
\left({ }^{\circ}\right)\end{array}$ & $\begin{array}{c}\text { Threshold } \\
\text { (TeV) }\end{array}$ & $\begin{array}{c}\text { Sensitivity } \\
>1 \mathrm{TeV} \\
(\% \text { Crab) }\end{array}$ \\
\hline H.E.S.S. & -23 & 16 & 1800 & 4 & 107 & 960 & 5 & 0.1 & 0.7 \\
\hline H.E.S.S. II & -23 & 16 & 1800 & 1 & 614 & 2048 & 3.2 & tbd & tbd \\
\hline VERITAS & 32 & -111 & 1275 & 4 & 106 & 499 & 3.5 & 0.07 & 0.7 \\
\hline MAGIC I+II & 29 & -18 & 2225 & 2 & 234 & 1039 & 3.5 & 0.03 & 0.8 \\
\hline CANGAROO-III & -31 & 137 & 160 & 3 & 57.3 & 427 & 4 & 0.4 & 15 \\
\hline Whipple & 32 & -111 & 2300 & 1 & 75 & 379 & 2.3 & 0.3 & 15 \\
\hline HEGRA & 29 & 18 & 2200 & 5 & 8.5 & 271 & 4.3 & 0.5 & 5 \\
\hline CAT & 42 & 2 & 1650 & 1 & 17.8 & 600 & 4.8 & 0.25 & 15 \\
\hline
\end{tabular}

Table 1: Properties of selected air-Cherenkov instruments, including three of historical interest (Whipple, HEGRA and CAT). Adapted from ref. [15]. Significances relate to a point-like source detectable at the $5 \sigma$ significance level in a $50 \mathrm{~h}$ observation.

All these instruments are almost exclusively used by the groups who built them, with only limited access for external observers and, initially, with no provision for open data access. Such a mode of operation was appropriate for current instruments, which detect a limited number of sources, and for which the analysis and interpretation still requires the expertise and detailed technical knowledge of the instrument. However, a different approach is essential for CTA, due to the expected large increase in the number of detectable objects and the required collaboration with scientists working in other wavelength ranges. Also, releasing data to a wider public and supporting their use are important for maximising the scientific return of an observatory.

Besides a wealth of high-energy astrophysics results, CTA will have a large discovery potential in key areas of astronomy, astrophysics and fundamental physics research. These include the study of the origin of cosmic rays and their impact on the constituents of the Universe, the investigation of the nature and variety of black hole particle accelerators, and inquiries into the ultimate properties of matter and physics beyond the Standard Model, e.g., by searching for dark matter and the effects of quantum gravity.

The design goal is a factor of ten improvement in sensitivity in the currently accessible energy domain of about $100 \mathrm{GeV}$ to some $10 \mathrm{TeV}$ and for an extension of the accessible energy range to well below $100 \mathrm{GeV}$ and up to more than $300 \mathrm{TeV}$. This ambitious aim can only be achieved with a combination of telescopes of different sizes, large ones for the lowest energies, medium ones for the core energy range and many small ones for the highest energies ${ }^{3}$. To achieve a substantially improved sensitivity at the highest energies, CTA requires a collection area of the order of $10 \mathrm{~km}^{2}$ which means spreading numerous

\footnotetext{
${ }^{3}$ Such a combination was first considered in $1992[16]$.
} 
telescopes over a large area.

CTA will advance the state of the art in astronomy at the highest energies of the electromagnetic spectrum in a number of decisive ways, all of which are unprecedented in this field:

- Worldwide integration: CTA will for the first time bring together the experience of virtually all groups worldwide working with IACTs, and being interested in the astrophysics at the highest energies.

- Performance of the instrument: CTA aims to provide full-sky coverage, via a southern and northern site, with unprecedented sensitivity, spectral coverage, angular, energy and timing resolution. The large number of telescopes also will allow for independent operation of subarrays, with sensitivity of current instruments or better, which are either pointed at one source or are staggered to cover a larger area of the sky. These observing modes provide a high degree of flexibility of operation.

- Operation as an open observatory: CTA will, for the first time in this field, be operated as a true observatory, open to a wider scientific community, and providing support for easy access and analysis of data. Data will be made publicly available and will be accessible through Virtual Observatory tools. Service to professional astronomers will be supplemented by outreach activities and interfaces to the data which are suitable for laypersons.

- Technical implementation, operation, and data access: While based on existing and proven techniques, the goals of CTA imply significant advances in terms of efficiency of construction and installation, in terms of the reliability of the telescopes, and in terms of data preparation and dissemination. Therefore, the CTA observatory is qualitatively different from earlier experiments and its increase in capability goes well beyond anything that could be achieved through an expansion or upgrade of the existing instruments.

\section{CTA Science Drivers and Performance Goals}

The aim of CTA is to make significant progress over the existing experiments in every respect of science. The core science themes $[10,11,17]$ are:

- Cosmic Rays: According to the most accepted scenario, Galactic cosmic rays are accelerated in supernova remnants, and both, Agile and Fermi, are now seeing the characteristic $\pi^{0}$ spectral signature $[18,19]$. During the acceleration and propagation also gamma rays will be produced by cosmic ray interactions. Therefore, CTA should be able to detect a population of SNRs emitting VHE gamma rays, from which considerable insight into cosmic ray acceleration and propagation is gained [20]. In addition, CTA should have the capability to search for "PeVatrons", young supernova remnants that can accelerate particles up to $\mathrm{PeV}$ (i.e. $10^{15} \mathrm{eV}$ ) energies and contribute to the high-energy cosmic rays, and that have so far gone undetected. Instruments of the current generation have shown that cosmic ray interactions with interstellar gas produce an observable gamma-ray flux from galaxies beyond our own. With CTA, the number of detectable galaxies should dramatically increase. This would allow the study of the connection between cosmic rays and star-formation processes in galaxies.

- Black Holes, Jets and the Star-Forming History of the Universe: Supermassive 
black holes in the centres of active galaxies produce powerful outflows that offer excellent conditions for particle acceleration in shocks. CTA aims to measure large samples of such active galaxies of various types to study particle acceleration and gamma-ray emission processes [21]. The observations of rather close-by radio galaxies can shed light on the formation of the jet and its connection to the central black hole properties. In addition, the observations of some of the most powerful and most distant sources, the quasars, can tell us about the galaxy and the star-formation history of the Universe, which is imprinted in the amount and energy distribution of the extragalactic background light. On their way from a quasar to Earth the VHE gamma rays interact with this light and are absorbed. For a reliable estimate of the amount of this light, a large sample of spectra of active galactic nuclei (AGN) needs to be measured, which CTA should provide with its largely increased sensitivity [22].

- The Nature of Dark Matter and Lorentz Invariance Violation: A major open question in modern physics is the nature of dark matter. The most popular candidates are weakly interacting massive particles (WIMPs). The annihilation of such particles should produce detectable gamma-ray signals. CTA will have a much larger potential for dark matter detection than the current generation of IACTs. Its extended energy range should allow WIMPs with lower masses to be characterised, while improved sensitivity over the entire energy range and superior energy resolution should increase the probability of detection of dark matter through the observation of features in a dark-matter-induced photon spectrum. A larger field of view (FoV) with a homogeneous sensitivity, as well as the improved angular resolution, should allow for much more efficient searches for extended sources and spatial anisotropies. If signatures of dark matter will appear in direct-detection experiments, gamma-ray observations may provide complementary information to identify its properties and mass. Moreover, high-mass dark matter candidates could be missed by future direct-detection experiments, while CTA should be sensitive in the mass region from below $100 \mathrm{GeV}$ up to $10 \mathrm{TeV}$ [23]. In addition, the improved energy coverage and resolution will make CTA an excellent experiment for other fundamental physics questions, such as searches for axion-like particles, effects of quantum gravity and other violations of Lorentz invariance.

The articles contained in this Special Issue of "Astroparticle Physics" discuss these and many other science themes in more detail [20, 21, 22, 23, 24, 25, 26, 27, 28].

The CTA Observatory should consist of two sites, one in the southern and one in the northern hemisphere, allowing full-sky coverage and, consequently, access to more potential gamma-ray sources, to rare source classes with only few sources per hemisphere, and to rare events, such as VHE-rich GRB or a supernova explosion. The southern site will cover the central part of the Galactic plane and see many nearby Galactic sources, some of which will produce $\mathrm{PeV}$ gamma rays. It will therefore be designed to have a very good sensitivity over the full energy range, in particular, to cover energies from tens of $\mathrm{GeV}$ to above $100 \mathrm{TeV}$. The northern site will see less of the of the central, rich part of the Galactic plane and, thus, coverage of the highest energies will not be as critical as for the southern site. 
The science drivers are translated into specific performance goals for the observatory. They include in particular:

- Sensitivity: CTA will be about a factor of 10 more sensitive than any existing instrument in its energy range. As a consequence it will, for the first time, allow detection and in-depth study of large samples of known source types, it will explore a wide range of classes of suspected gamma-ray emitters and be sensitive to possible new phenomena that lie beyond the sensitivity of current instruments. In its core energy range, from about $100 \mathrm{GeV}$ to several TeV, CTA will have milli-Crab (mCrab) sensitivity, i.e. a factor of $10^{3}$ below the flux of the strongest steady source of VHE gamma rays (the Crab nebula), and a factor of $10^{4}$ below the highest fluxes measured so far in bursts from transient sources.

This dynamic range will not only allow the study of weaker sources and of new source types, it will also reduce the selection bias in the classification of known source types.

- Energy range: Broad coverage of the electromagnetic spectrum is crucial for understanding the physical processes occurring in VHE sources. With a single facility, CTA is aiming to cover four orders of magnitude in energy, from a few tens of $\mathrm{GeV}$ to a few hundred $\mathrm{TeV}$, again a factor of 10 more than any existing instrument. Together with the much improved precision and lower statistical errors, this will enable astrophysicists to distinguish between key hypotheses such as the leptonic or hadronic origin of gamma rays from SNR. Moreover, the energy range and the improved resolution are important for the detection of line emission from dark matter clusters. With Fermi and CTA operating simultaneously, an unprecedented seamless coverage of more than seven orders of magnitude in energy can be achieved.

- Angular resolution: Current instruments are able to resolve extended sources, but they cannot probe the fine structures visible in other wavebands. In SNRs, for example, the measurement of the width of the gamma-ray emitting shell would provide sensitive constraints on the acceleration mechanism. By selecting a subset of gamma-ray induced cascades detected simultaneously by many of its telescopes, CTA can reach angular resolutions of better than 2 arc minutes for energies above $1 \mathrm{TeV}$, a factor of 5 better than the typical values for current instruments.

- Temporal resolution: With its large detection area, CTA can resolve flaring and time-variable emission on sub-minute time scales [25], which is currently out of reach. In gamma-ray emission from active galaxies, variability time scales probe the size of the emitting region. Current instruments have already detected flares varying on time scales of a few minutes, requiring a re-assessment of the phenomena in the vicinity of the supermassive black holes at the cores of active galaxies, and the jets emerging from them. CTA will also give new insights in (quasi-) periodic phenomena such as the emission from inner stable orbits around black holes or from pulsars and other objects, where frequent variations and glitches in period smear the periodicity when averaging over longer times [26].

Table 2 summarises the performance goals of CTA.

CTA's increased sensitivity and enlarged energy range open new observing regions for high-energy astrophysics. It will allow both the detailed study of a diverse range of known objects, and the serendipitous discovery of entirely new phenomena. Resulting from the 


\begin{tabular}{|c|c|c|}
\hline $\begin{array}{l}\text { Diff. Sensitivity } \\
\left(\mathrm{erg} \mathrm{cm}^{-2} \mathrm{~s}^{-1}\right)\end{array}$ & $\begin{array}{l}\text { at } 50 \mathrm{GeV} \\
\text { at } 1 \mathrm{TeV} \\
\text { at } 50 \mathrm{TeV}\end{array}$ & $\begin{array}{c}8 \times 10^{-12} \\
2 \times 10^{-13} \\
3 \times 10^{-13}(\mathrm{~S}) / 10^{-12}(\mathrm{~N})\end{array}$ \\
\hline $\begin{array}{l}\text { Collection Area } \\
\qquad\left(\mathrm{m}^{2}\right)\end{array}$ & $\begin{array}{l}\text { at } 1 \mathrm{TeV} \\
\text { at } 10 \mathrm{TeV}\end{array}$ & $\begin{array}{c}>10^{4} \\
>10^{6}(\mathrm{~S}) />5 \times 10^{5}(\mathrm{~N})\end{array}$ \\
\hline Angular Resolution & $\begin{array}{c}\text { at } 0.1 \mathrm{TeV} \\
>1 \mathrm{TeV}\end{array}$ & $\begin{array}{l}0.1^{\circ} \\
0.05^{\circ}\end{array}$ \\
\hline Energy Resolution & $\begin{array}{l}\text { at } 50 \mathrm{GeV} \\
>1 \mathrm{TeV}\end{array}$ & $\begin{array}{l}\leq 25 \% \\
\leq 10 \%\end{array}$ \\
\hline Field of View & $\begin{array}{l}\text { at } 0.1 \mathrm{TeV} \\
\text { at } 1 \mathrm{TeV} \\
>10 \mathrm{TeV}\end{array}$ & $\begin{array}{c}5^{\circ} \\
8^{\circ} \\
10^{\circ}\end{array}$ \\
\hline Sensitivity in FoV & at $1 \mathrm{TeV}$ flat out to & $>2.5^{\circ}$ \\
\hline Source Localisation & at $1 \mathrm{TeV}$ & 5" per axis \\
\hline Repointing Time & $\begin{array}{c}<0.1 \mathrm{TeV} \\
0.1-10 \mathrm{TeV}\end{array}$ & $\begin{array}{l}20 \mathrm{~s} \text { (goal), } 50 \mathrm{~s} \text { (max) } \\
60 \mathrm{~s} \text { (goal), } 90 \mathrm{~s} \text { (max) }\end{array}$ \\
\hline
\end{tabular}

Table 2: Performance goals for the CTA observatories. The sensitivity is given for 5 bins per decade. For the sensitivity and the collection area at high energies, separate values for the southern (S) and northern (N) arrays are given.

above performance goals, CTA will have two unique features: the ability to produce the deepest sky surveys at VHE energies [29], and to observe shortest-timescale phenomena.

Surveys are ideally unbiased, systematic, exploratory recordings of what sources exist, and are therefore critical for energy domains that are investigated for the first time. A ten-fold improvement in sensitivity in the core energy window of CTA would mean, for example, that CTA could carry out a Galactic plane survey as extensive as that of H.E.S.S in five years $(2000 \mathrm{~h})$, but with a uniform sensitivity of $3 \mathrm{mCrab}$, i.e. well below all current individual detections. Such a new survey would give access to many dozens of SNRs and pulsar wind nebulae allowing, for the first time, population studies of these objects in VHE gamma rays. Beyond the Galactic plane, a blind survey has never been conducted. CTA will reach sensitivities similar to the flux level of the faintest AGN currently detected at VHE in 30 min exposures and with 740 such pointings could chart a quarter of the sky [29]. Such a survey could uncover new, unexpected classes of extragalactic VHE gammaray emitters, provide constraints on dark matter annihilation in the $\mathrm{TeV}$ region and probe the diffuse Galactic emission.

Short-timescale variations (few-minutes) in VHE gamma rays have been observed from AGN and, tentatively, in Galactic binary systems. With CTA, timescales of less than a minute measured in flaring AGN jets can strongly constrain the size of the flaring region. Opening up these short timescales may also provide clues on the formation of relativistic outflows from highly magnetised binaries. The current $\mathrm{MeV}$ and $\mathrm{GeV}$ gammaray measurements have revealed flares from supposedly steady sources, such as the Crab nebula. CTA could reveal if such flares happen in pulsar wind nebulae also at the highest 
energies. Transients produced by accretion and related ejection have been observed in the radio and X-ray bands for many Galactic sources. CTA could reveal the gamma-ray behaviour of such phenomena. Another transient phenomenon to be observed with CTA is gamma-ray bursts (GRBs), since Fermi has already detected photons of a few tens of $\mathrm{GeV}$ from some GRBs (or up to $100 \mathrm{GeV}$ intrinsic energies, without the cosmological redshift) despite its much smaller effective area. The fast slewing capabilities and the low energy threshold of CTA make it very promising for GRB detection [28].

\section{CTA Technological Concept}

To reach the performance goals summarised in table 2, and specifically the wide energy range to be covered, the instrumentation needs to be optimised for three adjacent energy ranges (without strong overlap).

- The low-energy range $\leq \mathbf{1 0 0} \mathrm{GeV}$ : To detect showers down to a few tens of $\mathrm{GeV}$, the Cherenkov light needs to be sampled efficiently, with the fraction of area covered by light collectors being of the order of $10 \%$. Since event rates are high and systematic uncertainties of the background limit the achievable sensitivity, the area of this part of the array can be relatively small (of the order of a few $10^{4} \mathrm{~m}^{2}$ ). The CTA design assumes a small number $(\leq 4)$ of closely placed large-size telescopes (LSTs), with a mirror diameter of about $23 \mathrm{~m}$, to collect as many Cherenkov photons as possible from the low energy showers. These telescopes require the short repointing time quoted in table 2 to allow quick follow-ups of GRB alerts.

- The core energy range 0.1-10 TeV: Shower detection and reconstruction in this energy range are well understood from current instruments. The appropriate step for improved performance is an array of mid-sized telescopes (MSTs) with mirrors of about $12 \mathrm{~m}$ diameter and a spacing of about $100 \mathrm{~m}$. Improved sensitivity compared to existing instruments will be obtained both by the increased area covered by the array and by the higher quality of shower reconstruction, since individual showers will typically be stereoscopically imaged by a larger number of telescopes than in current few-telescope arrays. For the first time, array sizes will become much larger than the Cherenkov light pool of a shower, ensuring that images will be uniformly sampled across the light pool and that a number of images are recorded close to the optimum distance from the shower axis (about $70-150 \mathrm{~m}$ ), where the light intensity is large and intensity fluctuations are small. Also, the shower axis is viewed under a sufficiently large angle for efficient reconstruction of its direction. At H.E.S.S. or VERITAS, for example, events which are seen and triggered by all four telescopes provide significantly improved resolution and strongly reduced backgrounds, but they represent only a relatively small fraction of events. For CTA almost all events will be recorded at high quality. A further advantage of CTA is that an extended telescope grid operated with a two-telescope trigger condition will have a lower threshold than a small array, since there are always telescopes sufficiently close to the shower core. - The high-energy range $>\mathbf{1 0} \mathbf{~ T e V}$ : Here, the main limitation is the number of detected gamma-ray showers. Consequently, to achieve large improvement the array needs to cover an area of several square kilometres. At high energies the light yield of a shower is large, so that showers can be detected well beyond the $150 \mathrm{~m}$ radius of a typical Cherenkov 
light pool. Two options can be considered: either a large number of small telescopes with mirror areas of a few $\mathrm{m}^{2}$ and spacing matched to the size of the light pool (100-200 m), or a smaller number of larger telescopes with $10-20 \mathrm{~m}^{2}$ area which can see showers out to core distances of $\geq 500 \mathrm{~m}$, and can hence be either deployed with a spacing of several $100 \mathrm{~m}$, or in widely separated sub-clusters consisting of a few telescopes. Both implementations are called Small Size Telescopes (SSTs). While it is not immediately obvious which option offers the best cost/performance ratio at high energies, the sub-cluster concept with larger telescopes has the advantage of providing additional high-quality shower images towards lower energies, for impact positions near the sub-cluster.

Irrespective of the technical implementation details, the performance of a single Cherenkov telescope is primarily characterised by its light collection capability, i.e. the product of mirror area and the photon collection and detection efficiencies, by its FoV and by its pixel size, which limits the size of image features that can be resolved. The optical system of the telescope should obviously be able to achieve a point spread function matched to the pixel size. The electronics for signal capture and triggering should provide a bandwidth matched to the length of Cherenkov pulses of a few nanoseconds. The performance of an array is also dependent on the triggering strategy. Cherenkov emission from air showers has to be separated in real time from the high flux of night sky background photons, based on individual images and the stereoscopic combination of images from several telescopes. The raw data stream from Cherenkov telescopes is far too large to be recorded without any reduction.

Besides mirror area, the FoV is another important parameter of a telescope. A relatively large FoV is mandatory for the widely spaced telescopes of the high-energy array, since the distance of the image from the camera centre scales with the distance of the impact point of the air shower to the telescope. The optimum size of the FoV is not easy to determine. From the science point of view, a large FoV is highly desirable, since it allows (i) the detection of high-energy showers at large impact distance without image truncation, (ii) the efficient study of extended sources and of diffuse emission regions, and (iii) large-scale surveys of the sky and the study of clustered sources, e.g. in the band of the Milky Way. In addition, a larger FoV generally helps to improve the uniformity of the camera and to reduce background systematics. However, larger FoV for a given pixel size results in rapidly growing cost for larger numbers of photo-sensors and electronics channels. A large FoV also requires technically challenging telescope optics. With the current single-mirror optics and $\mathrm{f} / \mathrm{D}$ ratios in the range up to 1.2 , an acceptable point spread function is obtained out to radii of $4-5^{\circ}$. Larger FoVs with single-mirror telescopes require increased $\mathrm{f} / \mathrm{D}$ ratios, in approaching 2 for a $10^{\circ} \mathrm{FoV}$, which are mechanically difficult to realise since a large and heavy focus box needs to be supported at a long distance from the mirror. Also, the single-mirror optics solutions, which provide the best imaging, use the Davies-Cotton design, which in turn result in a time dispersion of the Cherenkov photons that seriously affects the trigger performance, once the mirror diameters exceed $15 \mathrm{~m}$ (for the typical $\mathrm{f} / \mathrm{D}$ ratios). An alternative solution is the use of dual-mirror optics. With non-spherical primary and secondary mirrors, good imaging over fields of up to $10^{\circ}$ diameter can be achieved, but the disadvantages are the increased cost and complexity, 
significant shadowing of the primary mirror by the secondary, and complex alignment issues for faceted primary and secondary mirrors. Large incidence angles of photons onto the camera, which is common in dual-mirror optics, affects the photo detection efficiency and may require baffling of stray light.

Therefore, the choice of the FoV requires that science gains, cost and increased complexity be carefully balanced. When searching for unknown source types which are not associated with non-thermal processes in other, well-surveyed wavelength domains, a large FoV helps, as several sources may appear in one pointing. This increases the effective observation time per source by a corresponding factor compared to an instrument that can look only at one source at a time. An instrument with CTA-like sensitivity is expected to detect of the order of 1000 sources. In the Galactic plane, one would always find multiple sources in a FoV. In extragalactic space, the average angular distance between (an estimated 500) sources would be about $10^{\circ}$, implying that even for the maximum conceivable FoVs the gain is modest, but not negligible. Even in the Galactic plane, a very large field of view will not be the most cost effective solution, since the gain in terms of the number of sources viewed simultaneously scales essentially linearly with the diameter of the field of view, given that sources are likely to cluster within a fraction of a degree from the Galactic plane, whereas camera costs scale with the diameter squared. A rough estimate based on typical mirror costs and per-channel pixel and readout costs suggests an economic optimum in the cost per source-hour at around a FoV of $6-8^{\circ}$.

Detailed studies related to dish and mirror technology and costs, and the per-channel cost of the detection system, justify the FoV and pixel size for the various telescope designs shown in figs. 1 to 5 .

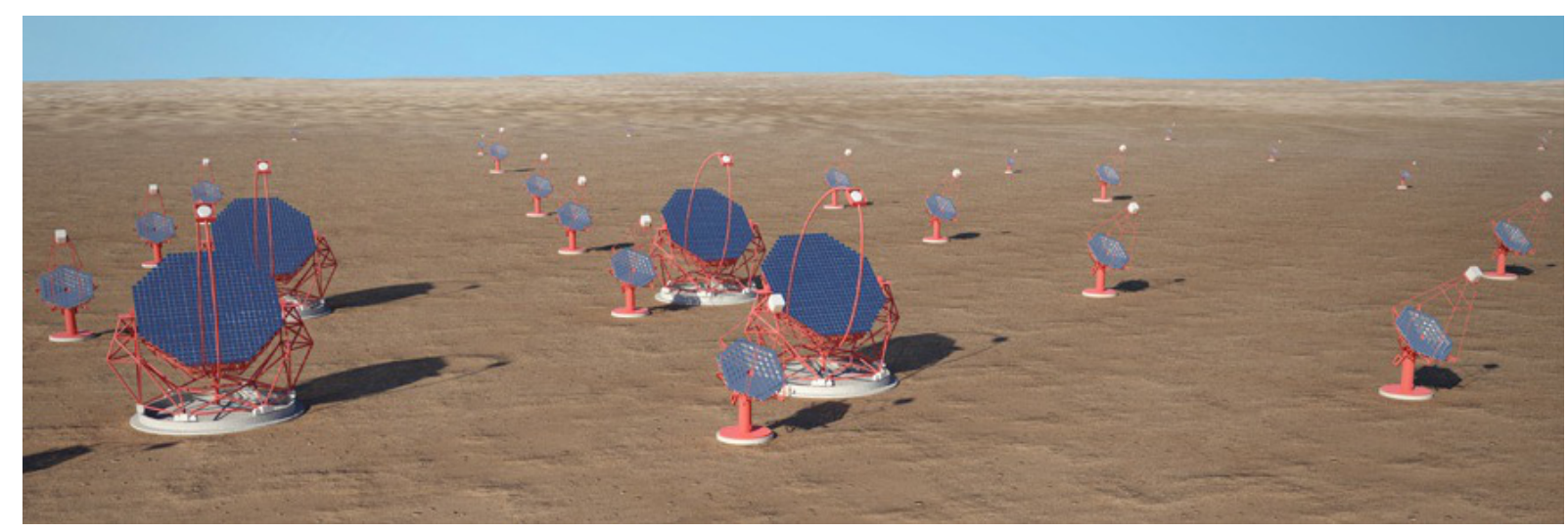

Figure 1: The basic CTA concept. Artist's view of the central part of a possible array configuration. Four LSTs, $\sim 30 \mathrm{MSTs}$, and $\sim 50 \mathrm{SSTs}$, at larger distances, scattered over several square kilometres.

The detailed design of these telescopes, their structures, reflectors and cameras, is largely based on well-proven technologies developed for the telescopes of H.E.S.S., MAGIC and VERITAS, yet, significantly improved in terms of reliability, availability, maintainability and safety (RAMS). Some novel design features are extensively tested and benefit greatly from the general experience gained in current projects. 
The main design drivers for these telescopes are the following:

LSTs: The desire to rapidly repoint the telescopes for rapid GRB follow-up motivates the choice of a light-weight structure of stiff carbon tubes holding a $23 \mathrm{~m}$ diameter reflector, similar to the MAGIC design. At most, four of these telescopes will be used in each CTA observatory. Their design is optimised to reach the best performance with lowest-possible energy threshold. The baseline design has a parabolic mirror with $27.8 \mathrm{~m}$ focal length, $4.5^{\circ} \mathrm{FoV}$ and $0.1^{\circ}$ pixels using PMTs (see fig. 2).

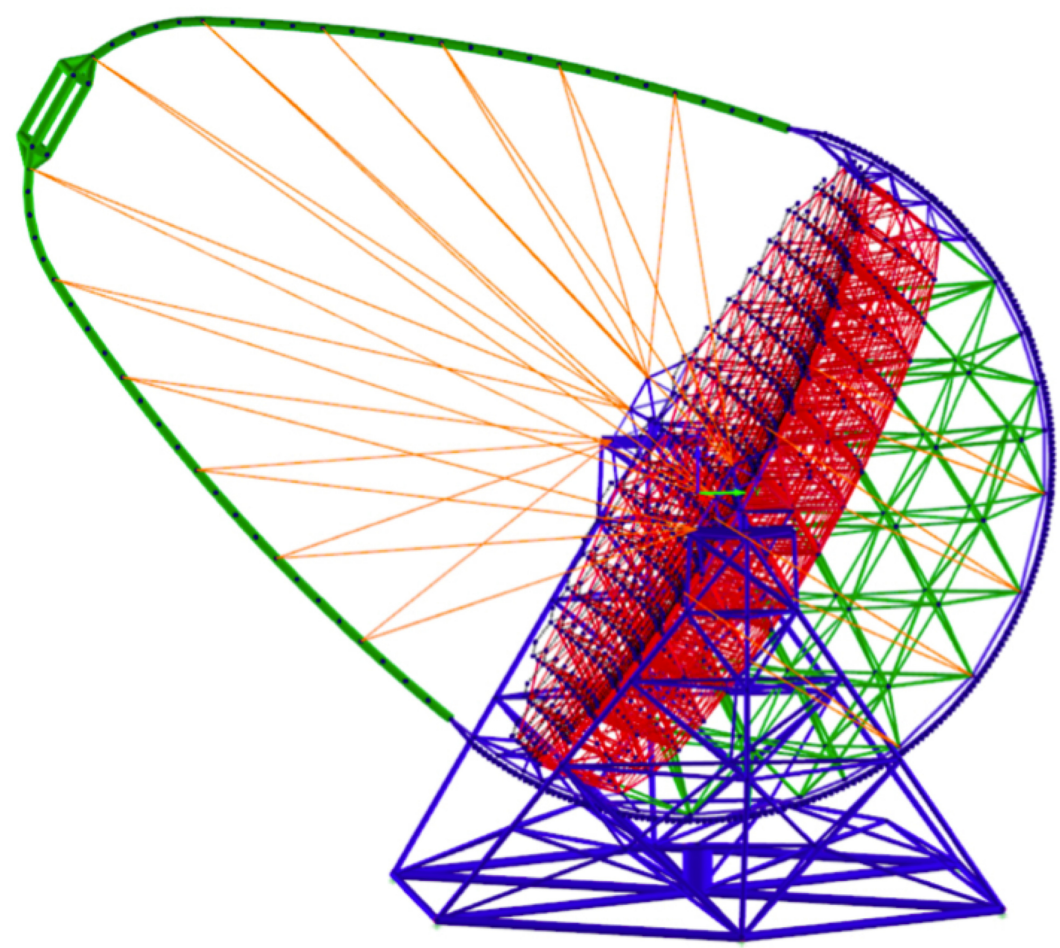

Figure 2: The baseline design for an LST of $23 \mathrm{~m}$ diameter, with $4.5^{\circ} \mathrm{FoV}$ and 2500 pixels of $0.1^{\circ}$ diameter.

MSTs: The MST design is a blend between the H.E.S.S. and VERITAS concepts for a $12 \mathrm{~m}$ diameter Davies-Cotton reflector, optimised for reliability, simplicity and costsaving, given that of the order of 30 such telescopes will be used at each site. The optical design foresees $16 \mathrm{~m}$ focal length, $7-8^{\circ} \mathrm{FoV}$ and $0.18^{\circ}$ pixels (fig. 3). Currently a fullscale prototype is under construction. In addition to these telescopes, CTA is exploring a design for a dual-mirror MST. This design might become a first extension of the southern CTA array, where as many as 36 telescopes could complement the baseline MST array. It has a Schwarzschild-Couder optics providing a $10^{\circ} \mathrm{FoV}$ and a very small plate scale. The latter allows for much finer pixelation and the use of much cheaper photo sensors (either multi-anode photomultiplier tubes or Silicon photomultipliers) in the camera. This is a completely new concept for IACTs and a prototype to prove its viability is being constructed (fig. 4). 


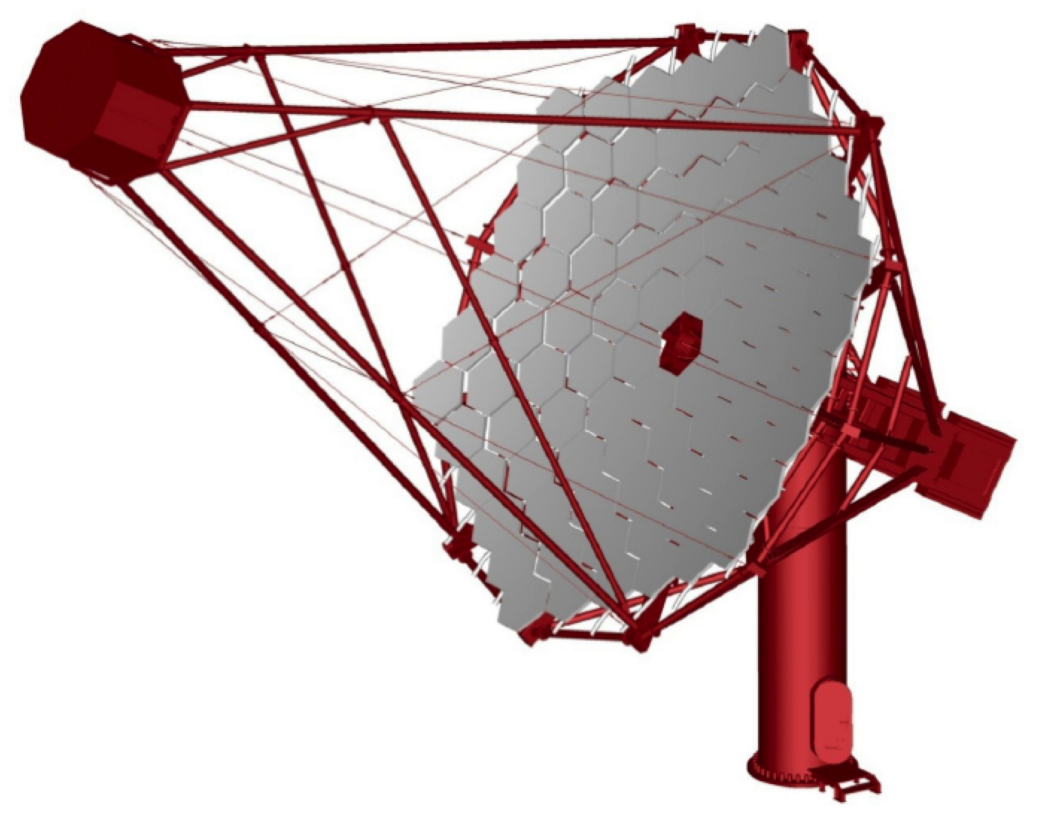

Figure 3: The baseline design for the $12 \mathrm{~m}$ diameter MST of Davies-Cotton type, with $8^{\circ}$ FoV and 1500 pixels of $0.18^{\circ}$.

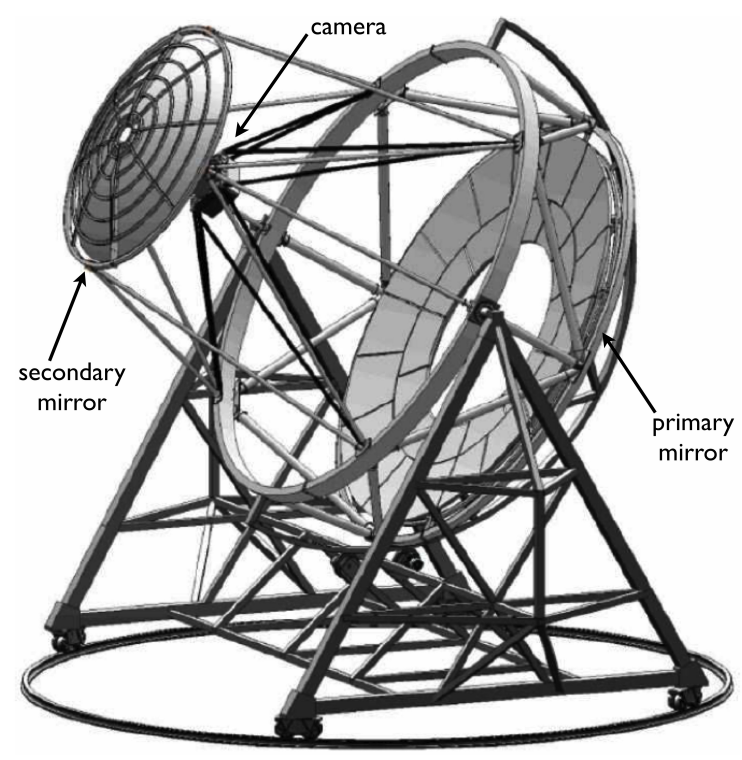

Figure 4: The design for a Schwarzschild-Couder dual-mirror MST, with a compact camera close to the secondary mirror. It will have a FoV of $8^{\circ}$ diameter, consisting of 11000 square pixels of $0.067^{\circ}$ side length.

SSTs: A rather large number (35-70, depending on cost) of small-size telescopes spread 
out over a large area are needed to reach the desired sensitivity at the highest energies. Therefore, the cost per telescope is one of the strongest drivers in the choice of the technology. In principle the SSTs could be designed as a simplified and downscaled version of the MSTs. However, the need for a large FoV due to the large inter-telescope spacing, would lead to the cost of the camera dominating the total SST cost. Therefore, different solutions are being explored (fig. 5). Possibilities are, for instance, the use of compact dual-mirror Schwarzschild-Couder (SC) optical design, with a very small plate scale (allowing for a small and thus inexpensive camera) or Davies-Cotton telescopes with cameras using the same new and inexpensive photosensor technologies that are proposed for the SC MST design. At present, different prototypes of both options are being developed to evaluate the feasibility and cost.

The advantages of Silicon-based detectors, such as their low power consumption, high photon detection efficiency and operational stability have recently been demonstrated with the FACT telescope [30].

Fig 1 shows a partial view of the central part of a possible CTA array configuration with the current designs for the LSTs, MSTs and SSTs. 


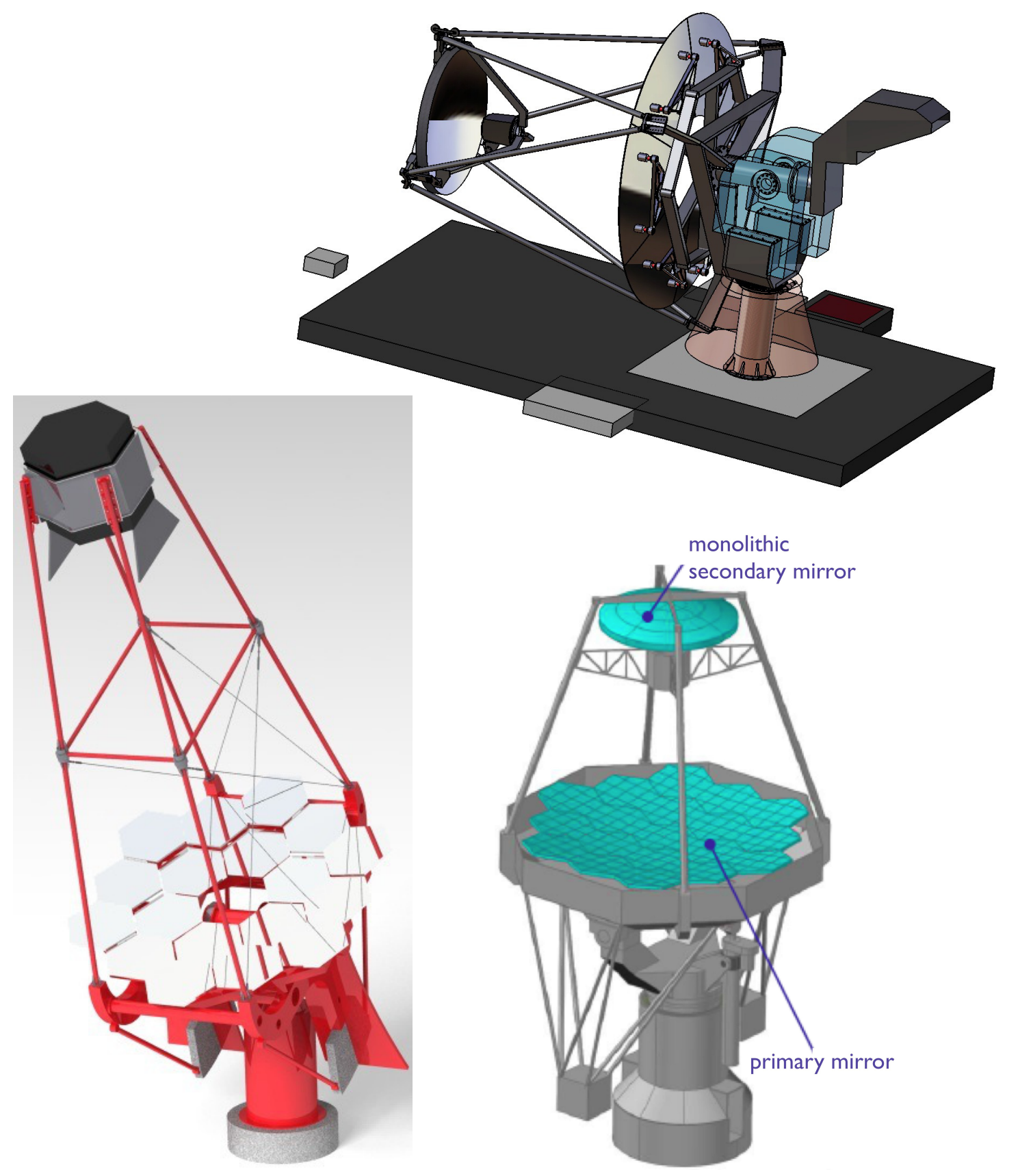

Figure 5: Three possible designs for SSTs of about $4 \mathrm{~m}$ mirror diameter, with $8-10^{\circ} \mathrm{FoV}$ and 1300 2000 pixels of $0.2-0.3^{\circ}$. Top and bottom right: Schwarzschild-Couder dual-mirror optics. Bottom left: Traditional Davies-Cotton design with $\mathrm{f} / \mathrm{D}=1.4$ and a large camera. 


\section{CTA Performance}

Determining the arrangement and characteristics of the CTA telescopes in the southern and northern arrays is a complex optimisation problem, requiring a balance of cost against performance in different bands of the spectrum. The article on Monte Carlo (MC) simulation in this issue [31] gives a detailed description of the layout and performance studies conducted so far for $\mathrm{CTA}^{4}$. Many candidate layouts have been considered. One promising configuration which is a good compromise between high-energy and low-energy performance was array E, which is shown on the right in fig. 6. The final array will likely look similar. Array E consists of three telescope types: 4 telescopes of $24 \mathrm{~m}$ diameter with $5^{\circ} \mathrm{FoV}$ and $0.09^{\circ}$ pixels, 23 telescopes of $12 \mathrm{~m}$ diameter with $8^{\circ} \mathrm{FoV}$ and $0.18^{\circ}$ pixels, and 32 telescopes of $7 \mathrm{~m}$ diameter with a $10^{\circ} \mathrm{FoV}$ and $0.25^{\circ}$ pixels.

For comparison also arrays B and C are shown. Array B has four LSTs in the centre and a compact array of MSTs surrounding it, but no SSTs. Consequently, it has a better performance than array $\mathrm{E}$ at low energies and a worse one at high energies. Array $\mathrm{C}$ consists only of MSTs which are positioned closer together in the centre and further apart away from the centre. A much larger area is covered by this array, and consequently it has a better high-energy performance than arrays B and E, yet its low-energy performance is worse.

The telescopes are distributed over $\sim 3 \mathrm{~km}^{2}$ on the ground and the effective collection area of the array at energies beyond $10 \mathrm{TeV}$ is considerably larger than this. The differential sensitivity of array E, derived from detailed MC calculations and standard data analysis techniques, is shown in fig. 7. For the northern CTA observatory the sensitivity at the highest energies will be reduced, due to the lack of SSTs. The figure illustrates the ranges in which each of the telescope types (LST, MST and SST) dominate the sensitivity, and the complementarity of the three telescope sizes to reach a seamless coverage of the whole CTA energy range.

As can be seen from fig. 8, such an array performs an order of magnitude better than other instruments over much of the target energy range. The figure shows the integral sensitivity estimated with MC simulations for CTA, together with the sensitivity in comparable conditions for some of the existing and future VHE gamma-ray installations. More sophisticated analyses are expected to give up to a factor of two better sensitivities.

The angular resolution of this array approaches 1 arc minute at high energies as can be seen in fig. 9, which displays the angular resolution from MC simulations for CTA, compared with the resolutions for some of the existing and future VHE gammaray installations. Events with many shower images, as they will be recorded with CTA, provide a clearly better resolution than events with only two images, which is the most common case for H.E.S.S. and VERITAS. In addition, the energy resolution of layout E is better than $10 \%$ above a few hundred $\mathrm{GeV}$.

\footnotetext{
${ }^{4}$ The first, large MC production used reasonable values for the various telescope parameters. For the second production, which is now in progress, the telescope specifications correspond more closely to the current base-line design.
} 


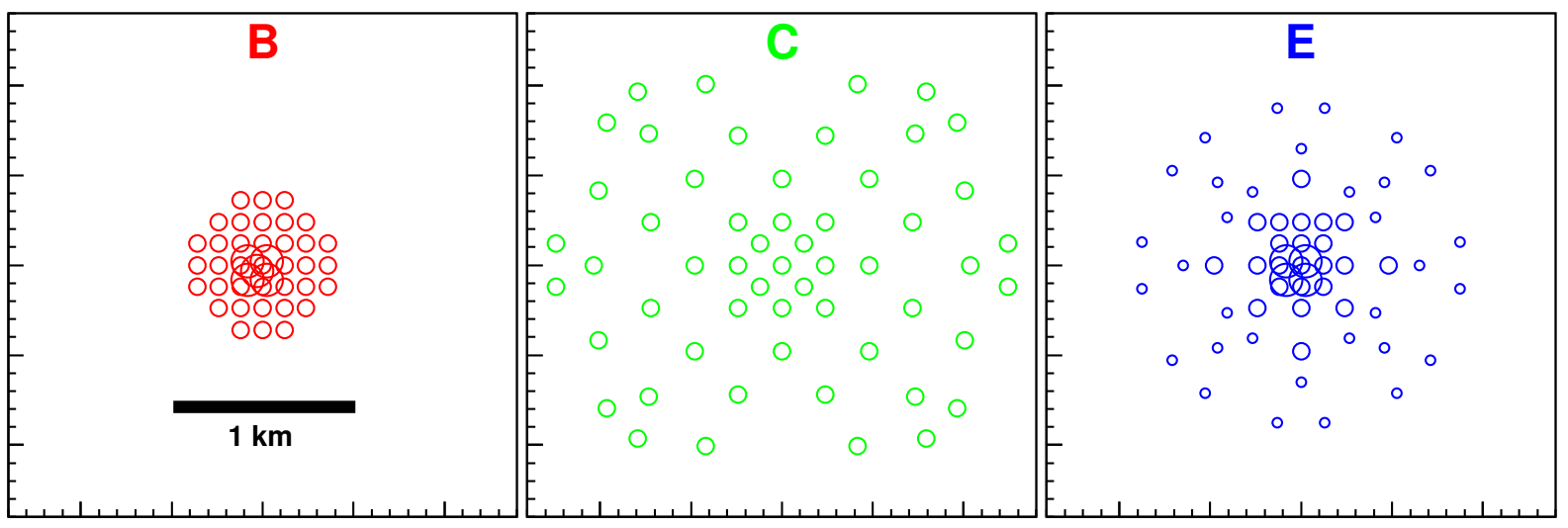

Figure 6: Different possible array layouts with estimated construction costs within the assumed budget. The circle sizes (not to scale) identify LSTs (large circles), MSTs (mid-size circles) and SSTs (small circles). The array with the most balanced performance in MC production 1 was array E.

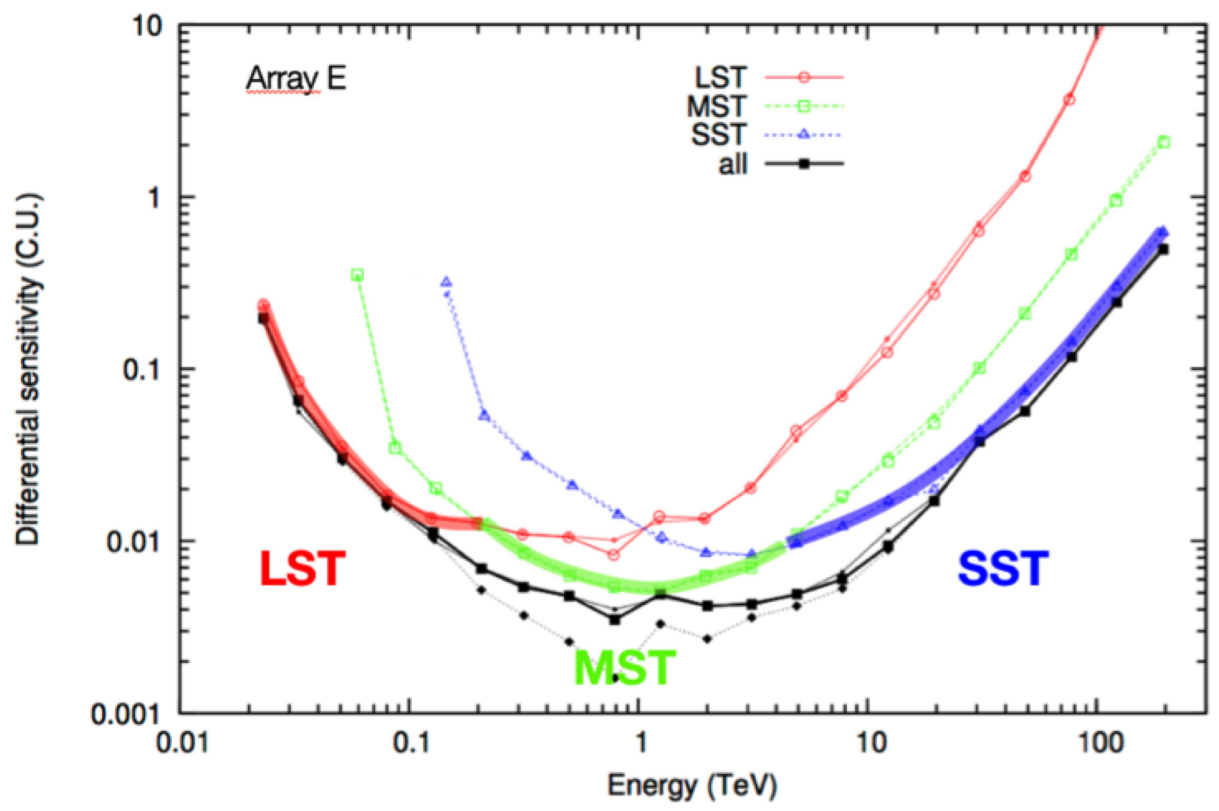

Figure 7: Differential sensitivity (in units of the energy-dependent flux of the Crab nebula) for array E ( $50 \mathrm{~h}, 5 \sigma, 5 \%$ background, 10 events, alpha=0.2, i.e. intervals of the decimal exponent of $0.2 \mathrm{meaning}$ 5 logarithmic bins per energy decade). Thin lines with small symbols illustrate the limited impact of a reduced dynamic range of the readout electronics (clipped at 1000 photoelectrons). The dashed black line with diamonds, shows the sensitivity if there was no electron background. 


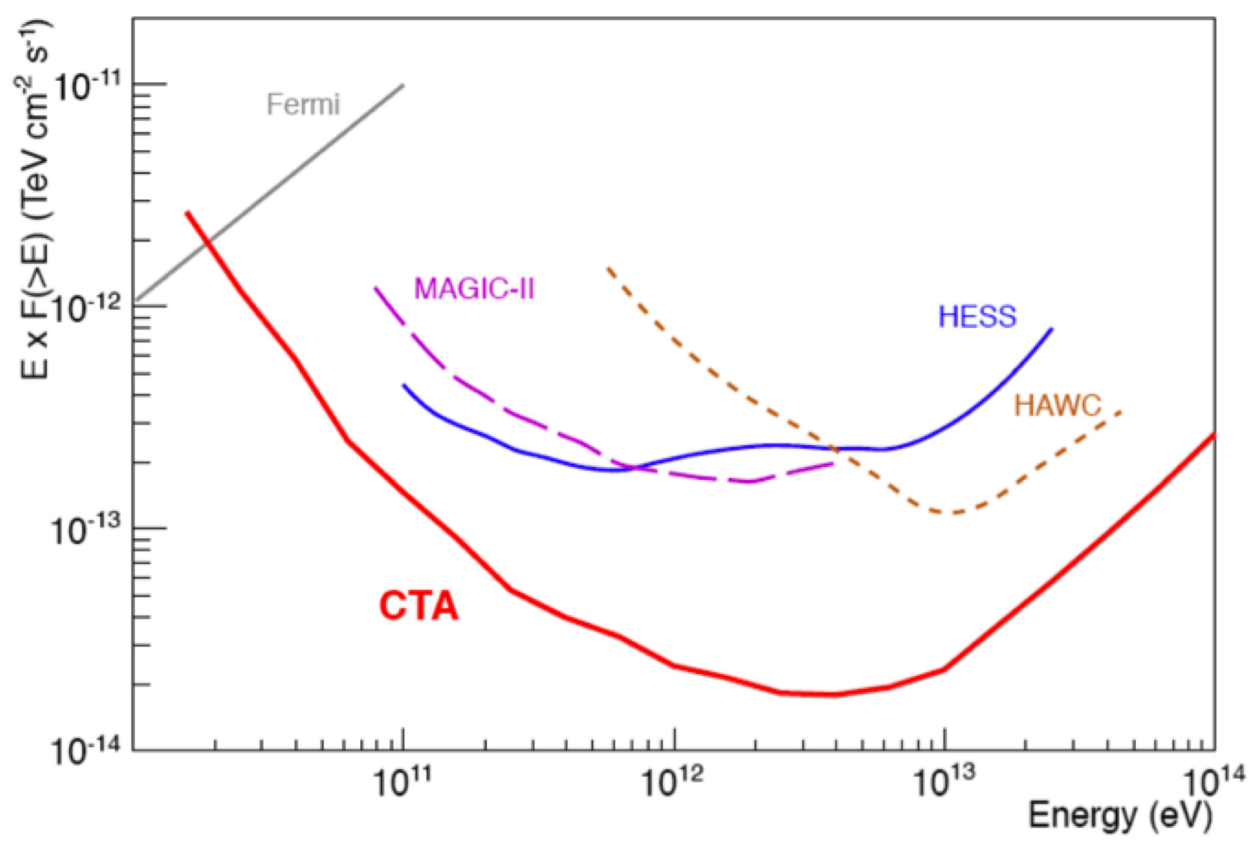

Figure 8: Integral sensitivity for CTA from MC simulations, together with the sensitivities in comparable conditions ( $50 \mathrm{~h}$ for IACTs, 1 year for Fermi-LAT and HAWC) for some gamma-ray observatories.

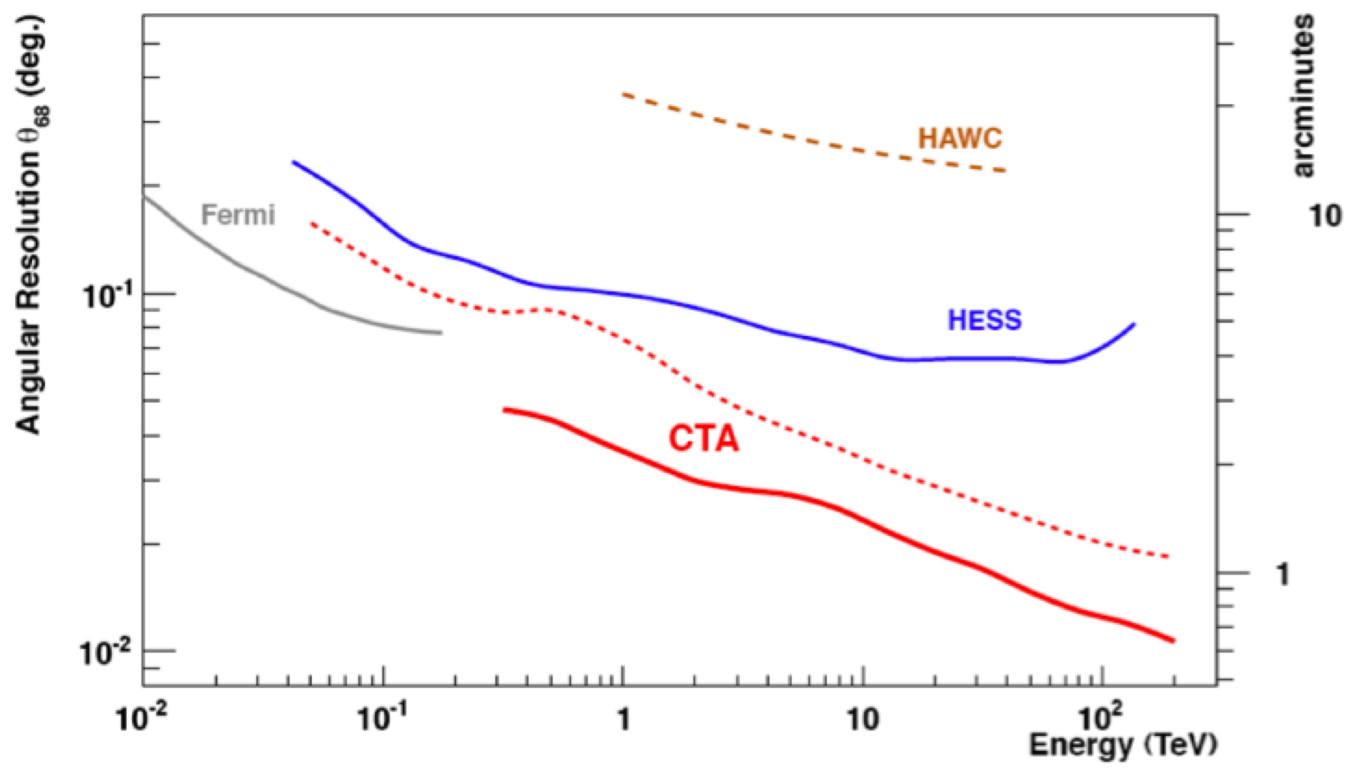

Figure 9: Angular resolution for CTA, compared with some existing and future VHE gamma-ray observatories. The solid line provides the angular resolution of CTA obtained from events with ten or more images, the dashed line shows the angular resolution for events with only two images. 
Array layout E has a nominal construction cost of $80 \mathrm{M} €$ (at 2006 cost levels) and meets the main design goals of CTA. Given that the configuration and the analysis methods used have not yet been fully optimised, it is likely that a significantly better sensitivity can be achieved, at nominal cost, with an array that follows basically this layout. Therefore, we are confident that the design goals of CTA can be realised within the envisaged cost, despite the uncertainties that are still present in the cost model.

\section{CTA as an Observatory}

CTA is to address a wide range of questions from astroparticle physics, astrophysics, cosmology and fundamental physics. Surveys will constitute a fundamental part of the core science program, which will include for example a survey of the Galaxy and deep observations of "legacy sources". As CTA will be operated as an open observatory, the scientific programme will be largely driven by observing proposals for individual sources which will be selected by peer-review for scientific excellence among suggestions received from the wider community.

The terms of access of scientists from outside the CTA Consortium and the countries who are funding the construction and operation of CTA are yet to be specified, but, as for other major astrophysical facilities, a fraction of the observing time will be open to the whole astrophysics community. In addition, it is planned that at some point the archive of all data from CTA will be made public without restrictions.

As for current Cherenkov telescope arrays, the actual observations will normally be conducted over an extended period, with several different projects being scheduled each night. Due to the size of CTA and various observing modes available, the operation of the array will be fairly complex. Therefore, CTA observations will not be conducted by the scientists who proposed the observation, but by a team of dedicated operators. CTA observatory operation involves proposal handling and evaluation, managing observations and data-flow, conducting maintenance and planning of upgrades.

\section{Observatory organisation}

The main elements that guarantee the smooth running of the CTA observatory are (i) the Science Operation Centre, which is in charge of the organisation of observations, (ii) the Array Operation Centre, which conducts the operation, monitors the telescopes and the atmosphere, and provides all calibration and environmental data necessary for the analysis, and (iii) the Science Data Centre, which provides and disseminates data and analysis software to the science community at large, using common astronomical standards and existing computing infrastructures. (see fig. 10). The high data rate of CTA and the large computing power required for data analysis, data transfer, distributed data storage, analysis and data access. demand dedicated resources, such as GEANT and EGI. The CTA observatories will be placed in remote locations. Thus, high-bandwidth networking is critical for remote diagnostics and speedy transfer of the data to well-connected data centres. CTA aims to support a wide scientific community, providing access to its scientific

data that is archived in a standardised way. As for other projects in astronomy, a "Virtual Observatory" will provide access to the data. 


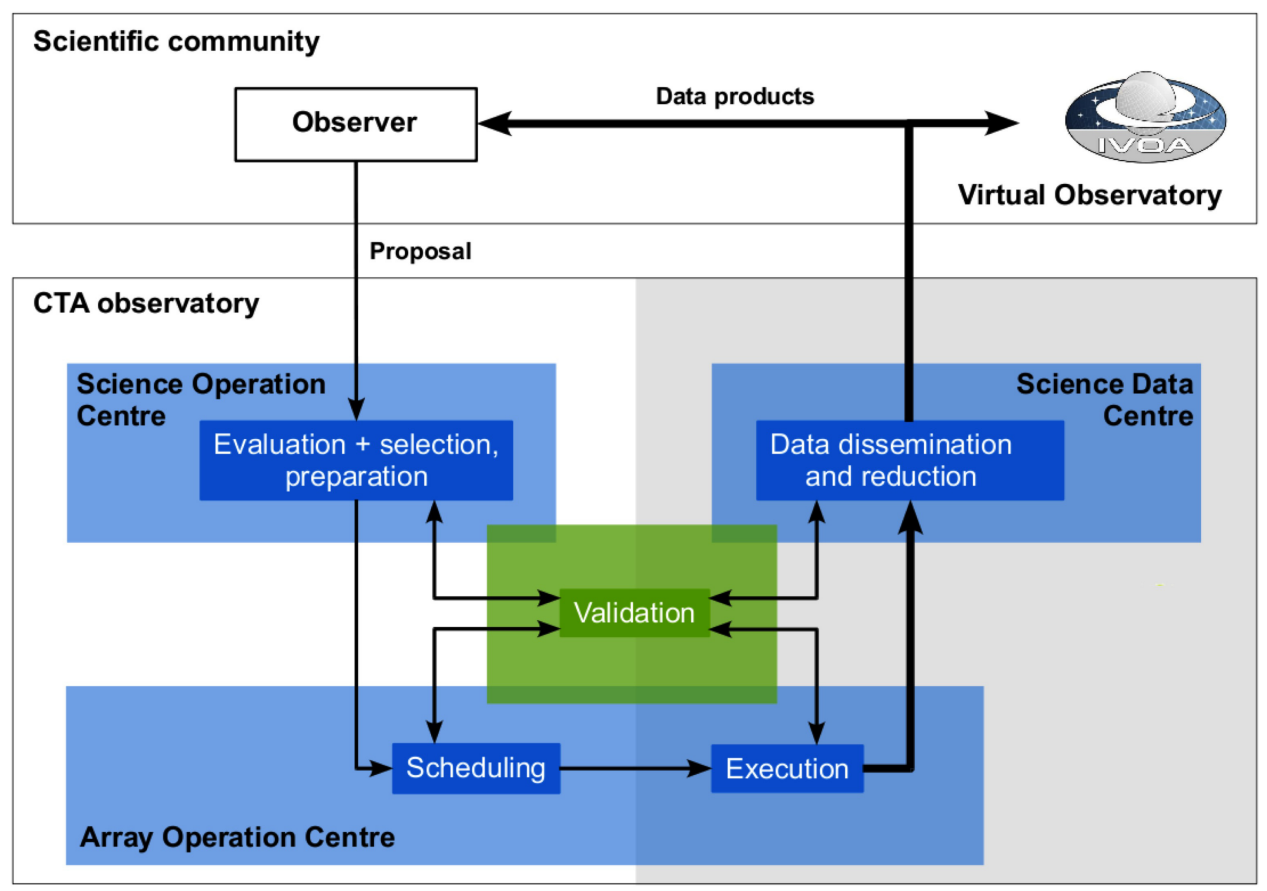

Figure 10: Organisation and work flow of the CTA observatory. The main elements are the Science Operation Centre, the Array Operation Centre and the Science Data Centre.

It is envisaged to start CTA operations during the construction phase, as soon as the first telescopes are ready to conduct competitive science observations.

\section{Proposal handling}

Observations with CTA are expected to serve a community of at least 1000 scientists, more than that of any national astronomical facility in Europe, and comparable to the size of the community using the ESO observatories in the 1980s. Therefore, CTA must deal efficiently with a large number of internal and external proposals for an observatory which is expected to be oversubscribed by a large factor. CTA plans to follow the practice of other major observatories and will announce calls for proposals at regular intervals. These proposals will be peer-reviewed by a group of international experts that will change on a regular basis. Different classes of proposals (targeted, surveys, coordinated multi wavelength campaigns, time-critical, target of opportunity, and regular programmes) are foreseen, as for current IACT experiments and ground-based observatories. Depending on the science under investigation, subarray operation may be required and each site can run several different observation programmes concurrently.

\section{Observatory operations}

Observatory operations covers day-to-day use of the arrays, observations and continuous hardware and software maintenance, proposal handling and evaluation, automated analysis and user support, as well as the long-term programme for upgrades and improvements to ensure continued competitiveness over the lifetime of the observatory. 
The observing programme of the CTA will be driven by the best proposals from the scientific community, selected in a peer-review process. The programme will be conducted in an automated fashion with a minimum number of observatory staff required on site. A quick pre-analysis will enable early checks and modification of observations, if necessary. After the observation, data and calibration files will be made available to the users.

Frequent modifications to the scheduled observing programme can be expected for several reasons. The transitory and variable nature of many of the phenomena to be studied requires flexible switching to flaring sources or bursts. CTA must adapt its schedule to changing atmospheric conditions. The possibility to pursue several potentially very different programmes at the same time increases the productivity of the CTA observatory. Routine calibrations and monitoring of the array and of environmental data must be scheduled as needed to ensure the required data quality.

\section{Data dissemination}

All measurements made with CTA will be subject to a rapid data analysis, including event selection and calibration of instrumental and environmental effects. The analysis of data obtained with Cherenkov telescopes differs from the procedures typical in other wavelength ranges in that extended MC simulations are needed to determine the effects of, and correct for, the influence of a large number of factors on the measurements. The necessary simulations will be carried out by CTA and will be used for the analysis of the data. Users will have access to the outputs of the standard data analysis and to the tools needed to use them. Storage of data and archiving of scientific and calibration data, programs, and MC simulations will be provided via distributed computing resources made available using the CTA EGI Virtual Organisation. The processing of CTA data is a major computational challenge. It will be necessary to reduce a volume of typically $10 \mathrm{~TB}$ of raw data per night to a few tens of MBytes of high-level data within a couple of hours. This first-level data processing will require hundreds of processors running in parallel on each site.

All levels of data will be archived in a standardised way, to allow access and reprocessing. Access to data, support services, software and data center infrastructures will be provided through a single access point, the "VHE gamma-ray Science Gateway". It is foreseen that individual scientists using the analysis software made available by CTA can conduct the high-level analysis of CTA data. This software will follow the standards used by other observatories. All aspects of data preparation, processing, dissemination and archiving will be organised and conducted by the science data centre.

\section{CTA Status and Plans}

CTA is the worldwide project for the future of VHE gamma-ray astronomy with Cherenkov Telescopes. It will consolidate VHE gamma-ray astronomy as one of the modern branches of astronomy. CTA is already considered one of the leading large astronomical observatories of this decade, together with ALMA [32], the E-ELT [33], SKA [34] and the LSST [35]. 
Currently, the CTA Consortium consists of over scientists and engineers from 27 countries from 5 continents and has become a truly global project.

Since 2008, CTA has been included in the roadmap of the European Strategy Forum on Research Infrastructures (ESFRI) [36]. It is one of the high-priority "Magnificent Seven" of the European strategy for astroparticle physics published by ASPERA [37], and is highly ranked in the "strategic plan for European astronomy" of ASTRONET [38]. In addition, CTA is a prioritised project for the current decade in the recently completed Decadal Survey of the US National Academy of Sciences [39].

The CTA Consortium started in 2007 to design the installation and to work towards its implementation. A Design Study phase has been ended in 2010 with the publication of a 120-page report [12]. CTA is now in the middle of a three-year EU funded Preparatory Phase, aimed to deliver a Technical Design Report and being "construction ready" towards the end of 2014. The five-year construction period could then be started in early 2015 (fig. 11).

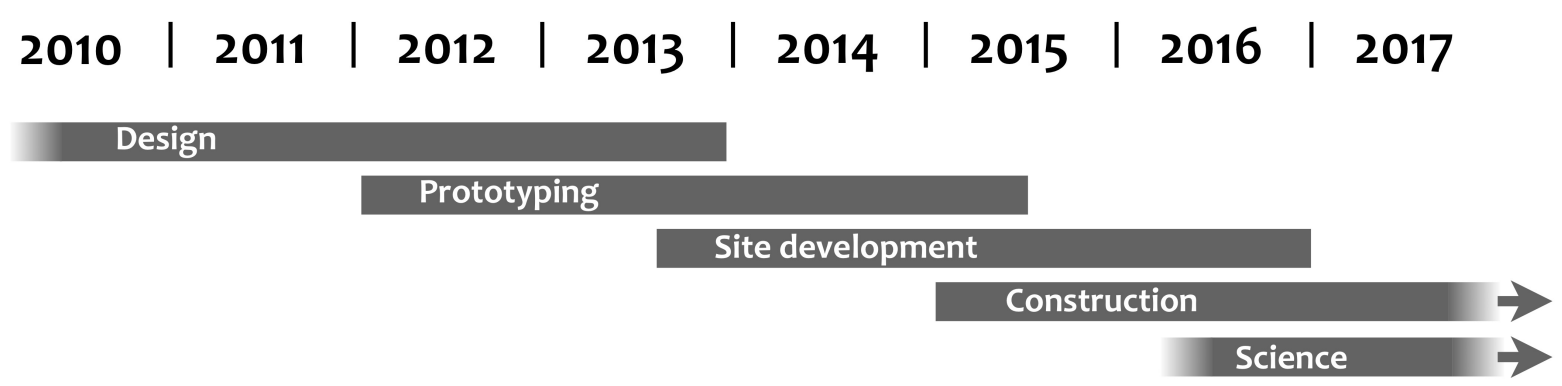

Figure 11: Current timeline of the CTA project.

An important milestone will be the selection of the location for the two CTA observatories. Ideal sites should provide a flat area of about $10 \mathrm{~km}^{2}$ at an altitude of $1.5-4.0$ $\mathrm{km}$ a.s.l., supreme astronomical sky conditions with minimum cloud cover and low levels of ambient light, easy access, and good local infrastructure. There are candidates for the southern site in Argentina, Namibia and Chile, and for the northern site on the Canary Islands, in Arizona, Mexico, China and India (see fig. 12). Currently, the suitability of all sites is investigated, and the site decision is anticipated in late 2013.

Funding agencies of the main countries participating in CTA have signed a "Declaration of Interest" in July 2012 to express their willingness to participate in the construction and operation of CTA. With their support it could be possible to start the scientific exploitation of the CTA observatories already by 2016, with a partial array, while the construction is still in progress. In just a few years, an exciting new view on the Universe at energies of up to $300 \mathrm{TeV}$ will emerge, and this new window will be pushed wide open. 


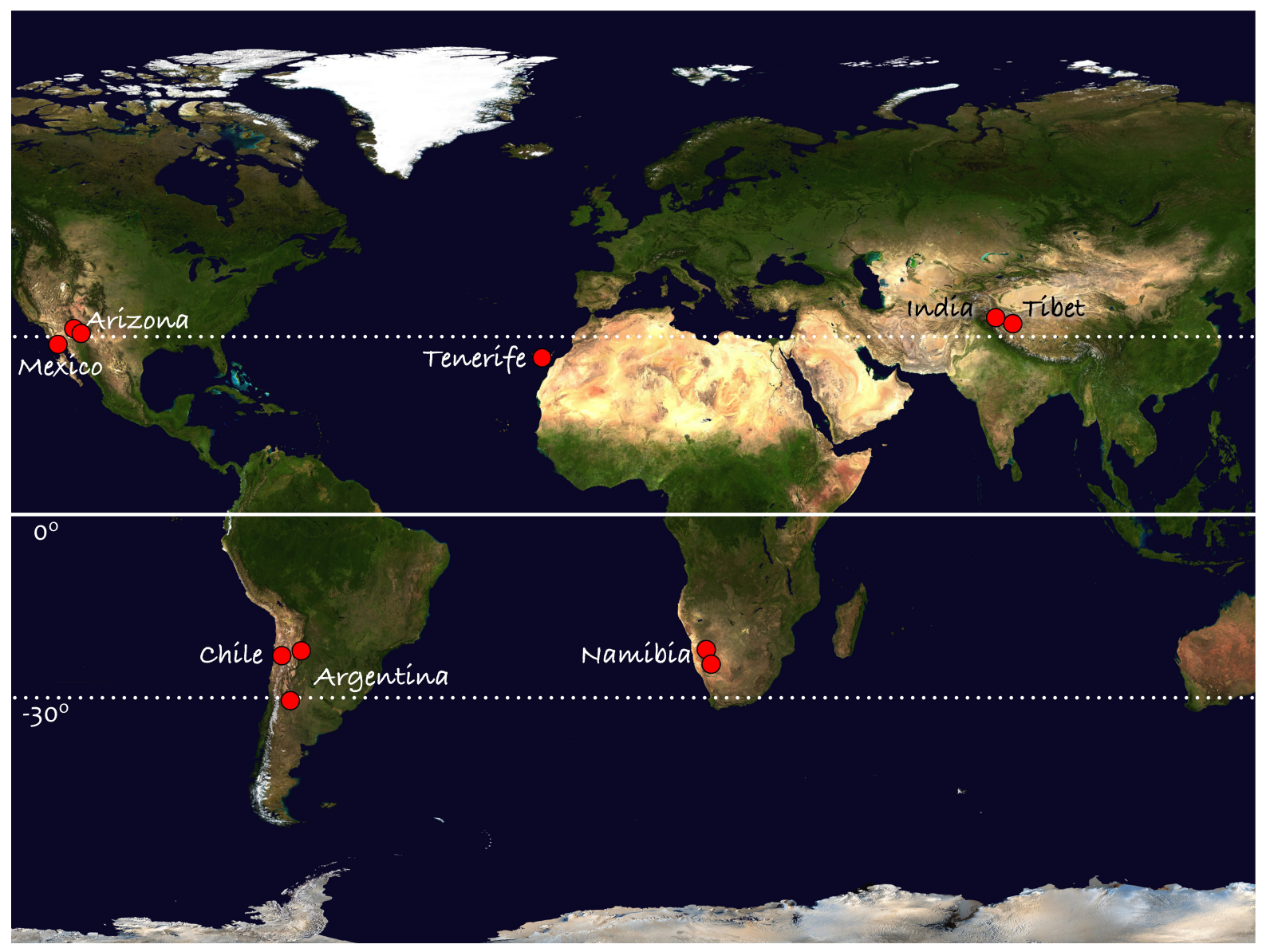

Figure 12: Approximate location of candidate sites in the range of $\pm 20-30^{\circ}$ latitude. 


\section{References}

[1] A.M. Hillas, Evolution of ground-based gamma-ray astronomy from the early days to the Cherenkov telescope arrays, Astropart. Phys. (2012), http://dx.doi.org/10.1016/j.astropartphys.2012.06.002

[2] F. Aharonian et al. (HESS Collaboration) A\&A 457 (2006) 899 http://www.mpi-hd.mpg.de/hfm/HESS/pages/about/telescopes

[3] MAGIC: http://magic.mppmu.mpg.de

[4] VERITAS: http://veritas.sao.arizona.edu

[5] W.B. Atwood et al., ApJ 697 (2009) 1071 doi:10.1088/0004-637X/697/2/1071 http://fermi.gsfc.nasa.gov

[6] M. Tavani et al., NIM A588 (2008) 52

[7] Milagro: http://www.lanl.gov/milagro

[8] Tibet AS Gamma: http://www.icrr.u-tokyo.ac.jp/em/index.html

[9] G. Aielli et al., Nucl. Instrum. Methods Phys. Res. A., 562 (2006) 92 http://argo.na.infn.it

[10] J.A. Hinton and W. Hofmann, Ann. Rev. of Astronomy and Astrophysics 47 (2009) 523

[11] F.A. Aharonian et al., Rep. on Prog. in Physics 71 (2008) 096901

[12] M. Actis et al. (CTA Consortium), Experimental Astronomy 32 (2011) 193-316 http://www.cta-observatory.org

[13] F. Aharonian et al. (HESS Collaboration), ApJ 636 (2006) 777-797. arXiv:0510397. http://dx.doi.org/10.1086/498013.

[14] HAWC: http://hawc.umd.edu

[15] J.A. Hinton, New Journal of Physics 11 (2009) 055005

[16] G. Fontaine, in "Towards a Major Atmospheric Cherenkov Detector", Editions Frontieres 1992.

[17] M. Martinez, J. Phys. Conf. Ser. 171 (2009) 012013

[18] A.A. Abdo et al., Science 327 (2010) 1103

[19] M Tavani et al., Ap. J. Lett. 710 (2010) L151

A. Giuliani et al., Ap. J. Lett. 742 (2011) L30

[20] F. Acero et al., Gamma-ray signatures of cosmic ray acceleration, propagation, and confinement in the era of CTA, Astropart. Phys. (2012), http://dx.doi.org/10.1016/j.astropartphys.2012.05.024 
[21] H. Sol et al., Active Galactic Nuclei under the scrutiny of CTA, Astropart. Phys. (2012), this issue

[22] D. Mazin et al., Potential of EBL and cosmology studies with the Cherenkov Telescope Array, Astropart. Phys. (2012), http://dx.doi.org/10.1016/j.astropartphys.2012.09.002

[23] M. Doro et al., Dark matter and fundamental physics with the Cherenkov Telescope Array, Astropart. Phys. (2012), http://dx.doi.org/10.1016/j.astropartphys.2012.08.002

[24] D. Dravins et al., Optical intensity interferometry with the Cherenkov Telescope Array, Astropart. Phys. (2012), http://dx.doi.org/10.1016/j.astropartphys.2012.04.017

[25] S. Funk, J.A. Hinton, Comparison of Fermi-LAT and CTA in the region between 10-100 GeV, Astropart. Phys. (2012), http://dx.doi.org/10.1016/j.astropartphys.2012.05.018

[26] E. de Oña-Wilhelmi et al., Prospects for observations of pulsars and pulsar wind nebulae with CTA,

Astropart. Phys. (2012), http://dx.doi.org/10.1016/j.astropartphys.2012.08.009

[27] J.M. Paredes et al., Binaries with the eyes of CTA,

Astropart. Phys. (2012), http://dx.doi.org/10.1016/j.astropartphys.2012.09.004

[28] S. Inoue et al., Gamma-Ray Burst Science in the Era of the Cherenkov Telescope Array, Astropart. Phys. (2012), this issue

[29] G. Dubus et al., Surveys with the Cherenkov telescope array, Astropart. Phys. (2012), http://dx.doi.org/10.1016/j.astropartphys.2012.05.020

[30] T. Bretz et al. (FACT Collaboration), FACT - The First G-APD Cherenkov Telescope (first results)", Proc. $5^{\text {th }}$ Int. Symp. on Gamma-Ray Astronomy, Heidelberg (2012)

[31] K. Bernlöhr et al., Monte Carlo design studies for the Cherenkov Telescope Array, Astropart. Phys. (2012),

http://dx.doi.org/10.1016/j.astropartphys.2012.10.002

[32] Alma: http://www.almaobservatory.org

[33] E-ELT: http://www.eso.org/public/teles-instr/e-elt.html

[34] SKA: http://www.skatelescope.org

[35] LSST: http://www.lsst.org

[36] ESFRI Roadmap: ftp://ftp.cordis.europa.eu/pub/esfri/docs/esfri_roadmap_update_2008.pdf

[37] ASPERA Roadmap: http://www.aspera-eu.org/images/stories/roadmap/aspera_roadmap.pdf

[38] ASTRONET Roadmap: http://www.astronet-eu.org/IMG/pdf/Astronet-Book.pdf

[39] Decadal Survey: http://sites.nationalacademies.org/bpa/BPA_049810 